\title{
Positive and necklace solitary waves on bounded domains
}

\author{
G. Fibich ${ }^{\mathrm{a}}$, D. Shpigelman ${ }^{\mathrm{b}}$ \\ ${ }^{a}$ School of Mathematical Sciences, Tel Aviv University, Tel Aviv69978 Israel, fibich@tau.ac.il \\ (corresponding author) \\ ${ }^{b}$ School of Mathematical Sciences, Tel Aviv University, Tel Aviv69978 Israel, dimashpi87@gmail.com
}

\begin{abstract}
We present new solitary wave solutions of the two-dimensional nonlinear Schrödinger equation on bounded domains (such as rectangles, circles, and annuli). These multi-peak "necklace" solitary waves consist of several identical positive profiles ("pearls"), such that adjacent "pearls" have opposite signs. They are stable at low powers, but become unstable at powers well below the critical power for collapse $P_{\mathrm{cr}}$. This is in contrast with the ground-state ("single-pearl") solitary waves on bounded domains, which are stable at any power below $P_{\mathrm{cr}}$.

On annular domains, the ground state solitary waves are radial at low powers, but undergo a symmetry breaking at a threshold power well below $P_{\mathrm{cr}}$. As in the case of convex bounded domains, necklace solitary waves on the annulus are stable at low powers and become unstable at powers well below $P_{\mathrm{cr}}$. Unlike on convex bounded domains, however, necklace solitary waves on the annulus have a second stability regime at powers well above $P_{\mathrm{cr}}$. For example, when the ratio of the inner to outer radii is 1:2, four-pearl necklaces are stable when their power is between $3.1 P_{\mathrm{cr}}$ and $3.7 P_{\mathrm{cr}}$. This finding opens the possibility to propagate localized laser beams with substantially more power than was possible until now.

The instability of necklace solitary waves is excited by perturbations that break the antisymmetry between adjacent pearls, and is manifested by power transfer between pearls. In particular, necklace instability is unrelated to collapse. In order to compute numerically the profile of necklace solitary waves on bounded domains, we introduce a non-spectral variant of Petviashvili's renormalization method.
\end{abstract}

Keywords: solitary waves; nonlinear chrodinger equation; bounded domains; nonlinear optics; stability

\section{Introduction}

The nonlinear Schrödinger equation (NLS) in free space

$$
\begin{gathered}
i \psi_{z}(z, x, y)+\Delta \psi+|\psi|^{2} \psi=0, \quad-\infty<x, y<\infty, \quad z>0, \\
\psi(0, x, y)=\psi_{0}(x, y), \quad-\infty<x, y<\infty
\end{gathered}
$$

is one of the canonical nonlinear equations in physics. In nonlinear optics it models the propagation of intense laser beams in a bulk Kerr medium. In this case, $z$ is the axial coordinate in the direction of propagation, $x$ and $y$ are the spatial coordinates in the transverse plane, $\Delta \psi:=\frac{\partial^{2}}{\partial x^{2}} \psi+\frac{\partial^{2}}{\partial y^{2}} \psi$ is the diffraction term, and $|\psi|^{2} \psi$ describes the nonlinear Kerr response

(C) 2015. This manuscript version is made available under the Elsevier user license http://www.elsevier.com/open-access/userlicense/1.0/ 
of the medium. For more information on the NLS in nonlinear optics and on NLS theory in free space and on bounded domains, see the recent book (1).

In some applications, it is desirable to propagate laser beams over long distances. In theory, this can be done by the NLS solitary waves $\psi_{\mathrm{sw}}=e^{i \mu z} R_{\mu}(x, y)$, where $R_{\mu}$ is a solution of

$$
\Delta R(x, y)-\mu R+|R|^{2} R=0, \quad-\infty<x, y<\infty .
$$

Unfortunately, the solitary wave solutions of (1) are unstable, so that when perturbed, they either scatter (diffract) as $z \rightarrow \infty$, or collapse at a finite distance $Z_{\mathrm{c}}<\infty$.

In order to mitigate this "dual instability" limitation, Soljacic, Sears, and Segev (2) proposed in 1998 to use a necklace configuration that consists of $n$ identical beams ("pearls") that are located along a circle at equal distances, such that adjacent beams are out of phase (i.e., have opposite signs), see Figure 1. The idea behind this setup is that the repulsion between adjacent out-of-phase beams resists the diffraction of each beam, and thus slows down its expansion. Necklace beams in a Kerr medium were first observed experimentally by Grow et al. (3). Necklace beams were also studied in $(4 ; 5 ; 6 ; 7)$. Recently, Jhajj et al. used a necklace-beam configuration to set up a thermal waveguide in air (8).

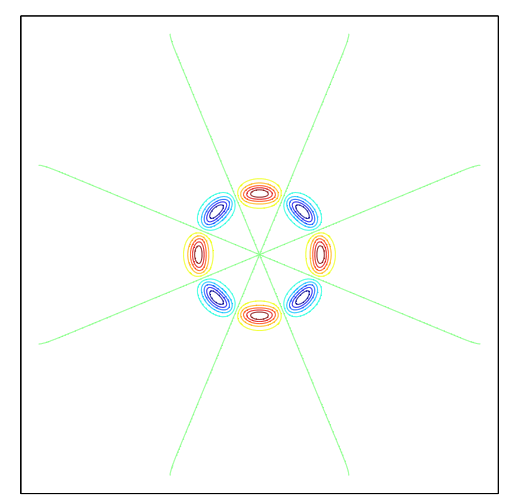

Figure 1: A circular necklace beam with 8 pearls (beams). Adjacent pearls are identical but have opposite phases. The electric field vanishes on the rays (solid lines) between adjacent pearls.

As we shall see, there are no necklace solitary wave solutions of the free-space NLS (1). Thus, in a bulk medium all necklace beams ultimately collapse or scatter. Yang et al. (7) showed theoretically and experimentally that solitary necklace solutions can exist in a bulk medium with an optically-induced photonic lattice. Because of the need to induce a photonic lattice, however, this approach is not applicable to propagation is a Kerr medium. In this study we show that necklace solitary waves exist in a Kerr medium, provided the beam is confined to a bounded domain. This setup corresponds to propagation in hollow-core fibers, and is therefore relatively easy to implement experimentally.

In hollow-core fibers, beam propagation can be modeled by the NLS on a bounded domain

$$
i \psi_{z}(z, x, y)+\Delta \psi+|\psi|^{2} \psi=0, \quad(x, y) \in D, \quad z>0
$$


subject to an initial condition

$$
\psi_{0}(0, x, y)=\psi_{0}(x, y), \quad(x, y) \in D
$$

and a Dirichlet boundary condition at the fiber wall

$$
\psi(z, x, y)=0, \quad(x, y) \in \partial D, \quad z \geq 0 .
$$

Here $D \subset \mathbb{R}^{2}$ is the cross section of the fiber, which is typically a circle of radius $\rho$, denoted henceforth by $B_{\rho}$.

Eq. (3) admits the solitary waves $\psi_{\mathrm{sw}}=e^{i \mu z} R_{\mu}(x, y)$, where $R_{\mu}$ is a solution of

$$
\begin{gathered}
\Delta R(x, y)-\mu R+|R|^{2} R=0, \quad(x, y) \in D, \\
R(x, y)=0, \quad(x, y) \in \partial D .
\end{gathered}
$$

In free space, the solitary-wave profile [i.e., the solution of (2)] represents a perfect balance between the focusing nonlinearity and diffraction. On a bounded domain, the reflecting boundary "works with" the focusing nonlinearity and "against" the diffraction. In fact, the reflecting boundary can support finite-power solitary waves even in the absence of a focusing linearity. These linear modes are solutions of the eigenvalue problem

$$
\Delta Q(x, y)=\mu Q, \quad(x, y) \in D, \quad Q(x, y)=0, \quad(x, y) \in \partial D
$$

Of most importance is the first eigenvalue of (5) and its corresponding positive eigenfunction, which we shall denote by $\mu_{\text {lin }}$ and $Q^{(1)}$, respectively.

Solitary wave solutions of (3) were studied by Fibich and Merle (9), Fukuizumi, Hadj Selem, and Kikuchi (10), and Noris, Tavares, and Verzini (11), primarily when $D$ is the unit circle $B_{1}$ and $R_{\mu}$ is radial, i.e., $R_{\mu}=R_{\mu}(r), r=\sqrt{x^{2}+y^{2}}$, and $0 \leq r \leq 1$. In that case, for any $\mu_{\text {lin }}<\mu<\infty$, there exists a unique positive solution $R_{\mu}^{(1)}(r)$. This solution is monotonically decreasing in $r$, and its power $P\left(R_{\mu}^{(1)}\right):=\int_{B_{1}}\left|R_{\mu}^{(1)}\right|^{2} d x d y$ is monotonically increasing in $\mu$ from $P=0$ at $\mu=\mu_{\text {lin }}+$ to $P=P_{\text {cr }}$ as $\mu \rightarrow \infty$. In addition to this ground state, there exist a countable number of excited radial states $\left\{R_{\mu}^{(n)}(r)\right\}_{n=2}^{\infty}$, which are non-monotone and change their sign inside $B_{1}$. These excited states have a unique global maximum at $r=0$, and additional lower peaks on concentric circles inside $B_{1}$.

The excited states $\left\{R_{\mu}^{(n)}(r)\right\}_{n=2}^{\infty}$ are the two-dimensional radial analog of the excited states of the one-dimensional NLS on an interval, see equation (26) below, which were studied by Fukuizumi et al. (10). In our study here we consider a different type of solitary waves of (3), which attain their global maximum at $n$ distinct points inside $D$. These necklace solitary waves are thus the non-radial two-dimensional analog of the one-dimensional excited states.

The paper is organized as follows. In Section 2 we briefly consider necklace solutions in $\mathbb{R}^{2}$, which correspond to propagation in a bulk medium. We illustrate numerically that their expansion is slower than that of single-beam solutions, and provide an informal proof that there are no necklace solitary waves in free space. In Section 3 we briefly review the theory for the NLS on bounded domains. In Section 4 we construct necklace solitary waves with $n$ pearls (peaks), denoted by $R_{\mu}^{(n)}$, on rectangular, circular, and annular domains. To do that, we first compute the single-pearl (single-peak/ground state) solitary wave of (4), 
denoted by $R_{\mu}^{(1)}$, on a square, a sector of a circle, and a sector of an annulus, respectively. Our numerical results for single-pearl solutions of (4) suggest that: ${ }^{1}$

1. $R_{\mu}^{(1)}$ exists for $\mu$ in the range $\mu_{\text {lin }}<\mu<\infty$.

2. As $\mu \rightarrow \mu_{\text {lin }}, R_{\mu}^{(1)}$ approaches the positive linear mode $Q^{(1)}$, i.e., $R_{\mu}^{(1)} \sim c(\mu) Q^{(1)}$, where $c(\mu) \rightarrow 0$.

3. As $\mu$ increases, $R_{\mu}^{(1)}$ becomes more localized, the effect of the nonlinearity becomes more pronounced, and that of the reflecting boundary becomes less pronounced.

4. In particular, as $\mu \rightarrow \infty, R_{\mu}^{(1)}$ approaches the free-space ground state $R_{\mu, 2 \mathrm{D}}^{(1) \text {,free }}$, which is the positive solution of $(2)$.

5. The pearl power $P\left(R_{\mu}^{(1)}\right):=\int\left|R_{\mu}^{(1)}\right|^{2} d x d y$ is monotonically increasing in $\mu$. In particular,

$$
\frac{d}{d \mu} P\left(R_{\mu}^{(1)}\right)>0, \quad \lim _{\mu \rightarrow \mu_{\mathrm{lin}}} P\left(R_{\mu}^{(1)}\right)=0, \quad \lim _{\mu \rightarrow \infty} P\left(R_{\mu}^{(1)}\right)=P_{\mathrm{cr}},
$$

where

$$
P_{\text {cr }}=\int_{\mathbb{R}^{2}}\left|R_{\mu, 2 \mathrm{D}}^{(1), \text { free }}\right|^{2} d x d y
$$

is the critical power for collapse.

6. On an annular domain, the ground state solitary waves are radial (ring-type) at low powers, but undergo a symmetry breaking into a single-peak profile at a threshold power well below $P_{\text {cr. }}$ In particular, equation (4) on the annulus,

(a) does not have a unique positive solution.

(b) has a positive solution which is not a ground state.

Similar results were obtained by Kirr et al. (12) for the NLS-GP equation with an inhomogeneous (e.g., double-well) potential.

Once we obtain the single-pearl profile, we construct the necklace solitary wave $R_{\mu}^{(n)}$ from $n$ identical pearls, such that adjacent pearls have opposite signs. Therefore, by construction,

$$
P\left(R_{\mu}^{(n)}\right)=n P\left(R_{\mu}^{(1)}\right)
$$

Hence, by (6),

$$
\frac{d}{d \mu} P\left(R_{\mu}^{(n)}\right)>0, \quad \lim _{\mu \rightarrow \mu_{\operatorname{lin}}} P\left(R_{\mu}^{(n)}\right)=0, \quad \lim _{\mu \rightarrow \infty} P\left(R_{\mu}^{(n)}\right)=n P_{\mathrm{cr}} .
$$

In Section 5 we study the stability of necklace solitary waves on bounded domains, by (i) computing the eigenvalues and eigenvectors of the associated linearized problem, and (ii) by solving the NLS with an initial condition which is a perturbed necklace solitary wave. These simulations show that:

1. Single-pearl (ground state) solitary waves $\psi_{\mathrm{sw}}^{(1)}=e^{i \mu z} R_{\mu}^{(1)}$ on bounded domains are stable for $\mu_{\text {lin }}<\mu<\infty$, i.e., for $0<P<P_{\text {cr }}$.

\footnotetext{
${ }^{1}$ These results are consistent with those obtained for radial positive solitary waves on the circle (9) and for positive one-dimensional solitary waves on an interval (10).
} 
2. For $n \geq 2$, there exists $\mu_{\mathrm{c}}$, where $\mu_{\text {lin }}<\mu_{\mathrm{c}}<\infty$, such that the necklace solitary waves $\psi_{\mathrm{sw}}^{(n)}=e^{i \mu z} R_{\mu}^{(n)}$ are stable for $\mu_{\text {lin }}<\mu<\mu_{\mathrm{c}}$, i.e., for

$$
0<P\left(R_{\mu}^{(n)}\right)<P_{\mathrm{th}}^{\text {necklace }}(n), \quad P_{\mathrm{th}}^{\text {necklace }}(n):=P\left(R_{\mu_{\mathrm{cr}}}^{(n)}\right),
$$

and unstable for $0<\mu-\mu_{\mathrm{c}} \ll 1$. Thus, the low-power necklace solitary waves inherit the stability of the linear necklace modes $e^{i \mu_{\operatorname{lin}} z} Q^{(n)}$ from which they bifurcate. ${ }^{2}$

3. On convex domains such as rectangles and circles, necklace solitary waves are unstable for $\mu_{\mathrm{c}}<\mu<\infty$, i.e., for

$$
P_{\text {th }}^{\text {necklace }}(n)<P\left(R_{\mu}^{(n)}\right)<n P_{\text {cr }}
$$

This may seem surprising, since $R_{\mu}^{(n)}$ satisfies the Vakhitov-Kolokolov (VK) condition for stability $\frac{d}{d \mu} P\left(R_{\mu}\right)>0$, see (8). We note, however, that the VK condition implies stability for ground-state solitary waves, which is not the case for necklace solitary waves. Indeed, the VK condition tests whether the solitary wave is susceptible to an amplitude (focusing) instability, whereby the solitary wave amplitude increases (decreases) as its width decreases (increases). The VK condition, however, does not determine whether the solitary wave is susceptible to other types of instabilities. See $(13 ; 14 ; 15)$ for more details.

4. The threshold power for necklace instability is substantially smaller than the critical power for collapse, i.e.,

$$
P_{\text {th }}^{\text {necklace }}(n)<P_{\text {cr. }} \text {. }
$$

Thus, the necklace becomes unstable when the power of each pearl is below $P_{\text {cr }} / n$. For example, the threshold power for instability of a necklace solitary wave with $n=4$ pearls is $\approx 0.55 P_{\mathrm{cr}}$ on a square domain and $\approx 0.24 P_{\mathrm{cr}}$ on a circular domain, i.e., when the power of each of the four pearls is roughly $0.14 P_{\mathrm{cr}}$ and $0.06 P_{\mathrm{cr}}$, respectively. As noted, single-pearl solitary waves are stable for $0<P<P_{\mathrm{cr}}$. Hence, power-wise, necklace solitary waves on circular and rectangular domains are considerably less stable than single-pearl solitary waves on these domains.

5. Necklace solitary waves on annular domains are also stable for $\mu_{\text {lin }}<\mu<\mu_{\mathrm{c}}$ and unstable for $0<\mu-\mu_{\mathrm{c}} \ll 1$. In addition, $P_{\mathrm{th}}^{\text {necklace }} \approx 0.24 P_{\mathrm{cr}}$ for a 4 -pearl necklace solitary waves on an annular domain with a 1:2 ratio of the inner to outer radii, which is the same as for a circular necklace. Thus, the addition of a hole "fails" to increase the threshold power for necklace instability.

Unlike on convex domains, however, necklace solitary waves on the annulus have a second stability regime at powers well above $P_{\mathrm{cr}}$. For example, when the ratio of the inner to outer radii is 1:2, 4-pearl necklaces are stable for powers between $3.1 P_{\mathrm{cr}}$ and $3.7 P_{\text {cr. }}$.

6. The instability of necklace solitary waves on circular, rectangular, and annular domains share many similar features. Thus, the unstable modes are those which are symmetric with respect to one or more interfaces between pearls. These modes break the antisymmetry between pearls, and thus allow for power transfer between pearls. Another

\footnotetext{
${ }^{2}$ Here $Q^{(n)}$ denotes a linear necklace solitary wave made out of $n$ identical $Q^{(1)}$ single pearls.
} 
common feature is that the unstable modes for a necklace with $2 \times 2$ pearls are either symmetric with respect to all four interfaces between pearls, or are symmetric in one direction and anti-symmetric in the perpendicular direction. In the latter case, the unstable modes consist of two identical unstable modes of a 2-pearl necklace on half the domain.

7. In Section 5.6 we consider the stability of necklace solitary waves of one-dimensional cubic and quintic NLS. We observe that the (in)stability properties of necklace solitary waves in the subcritical and critical cases are similar, in the sense that the instability occurs above a certain power threshold, and is related to power transfer between pearls. In particular, this further shows that necklace instability is unrelated to collapse.

In Section 6 we discuss the numerical methods. In particular, we introduce a novel non-spectral variant of Petviashvili's renormalization method that can be used to compute multi-dimensional solitary waves on bounded domains.

There are several unexpected results in this study:

1. The threshold power for instability of a necklace on a rectangular domain is twice that on a circular domain.

2. The threshold power for instability of a necklace on a circular domain is the same as that of an annular domain. This is surprising, since the hole reduces the interaction between the pearls, and so could be expected to have a stabilizing effect on the necklace.

3. In the case of necklace solitary waves on the annulus, there exists a second stability regime at powers above $3 P_{\mathrm{cr}}$.

4. To the best of our knowledge, our results for an annular domain provide the first example of a positive solitary wave of the homogeneous two-dimensional cubic NLS which

(a) satisfies the VK condition for stability, yet is unstable,

(b) is not a ground state, and

(c) is not the unique positive solitary wave.

As noted, such results were observed for the NLS-GP equation with an inhomogeneous potential (12).

The possibility to propagate stable necklace solitary waves with powers well above $P_{\text {cr }}$ may be important for applications. While an annular domain does not correspond to a standard hollow-core fiber, the NLS on an annular domain models beam propagation in a step-index optical fiber with a linear index of refraction

$$
n_{0}= \begin{cases}n_{0}^{(1)}, & \text { if } 0 \leq r<r_{1}, \\ n_{0}^{(2)}, & \text { if } r_{1}<r<r_{2}, \\ n_{0}^{(3)}, & \text { if } r_{2}<r<r_{3},\end{cases}
$$

where $0<r_{1}<r_{2}<r_{3}$ and $n_{0}^{(1)}, n_{0}^{(3)}<n_{0}^{(2)}$.

Our results can be extended in several directions. For example, NLS necklaces can be constructed on "irregular" two-dimensional domains, see e.g., Figure 5. Such necklace solitary waves may also be stable at powers above $P_{\text {cr. }}$. One can also construct necklace solitary waves in dimensions higher than 2. For example, one can compute a single-pearl 
solitary wave of the three-dimensional NLS on the unit cube, and then use it to construct a necklace solitary wave on a three-dimensional box. The question whether the NLS admits necklace solitary waves made out of non-identical pearls is open.

A note on notations. In this study we denote the profile of a necklace solitary wave with $n$ peaks (pearls) by $R_{\mu}^{(n)}$. In order to be consistent with this notation:

1. The single-pearl profile, i.e., the positive (ground state) solution of (4) is denoted by $R_{\mu}^{(1)}$, in contrast to the general convention of denoting the ground state by $R_{\mu}^{(0)}$.

2. The free-space necklace solutions in Section 2 are parameterized by $n=2 m$.

\section{Necklace solutions in free space}

The free space linear Schrödinger equation

$$
i \psi_{z}(z, x, y)+\Delta \psi=0, \quad-\infty<x, y<\infty, \quad z>0
$$

models the propagation of laser beams in a bulk linear medium. This equation admits the Laguerre-Gaussian vortex solutions

$$
\psi_{\mathrm{LG}}^{\mathrm{vortex}}(z, r, \theta):=\frac{1}{L(z)}\left(\frac{r}{L(z)}\right)^{m} e^{-\frac{1-4 i z}{L^{2}(z)} r^{2}+i(m+1) \zeta(z)} e^{i m \theta}, \quad m=1,2, \ldots,
$$

where $L(z)=\sqrt{1+16 z^{2}}, r=\sqrt{x^{2}+y^{2}}$, and $\zeta=-\arctan (4 z)$. Since $\Delta \psi=\psi_{r r}+\frac{1}{r} \psi_{r}+$ $\frac{1}{r^{2}} \psi_{\theta \theta}$, if $\psi=A(z, r) e^{i m \theta}$ is a solution of (9), then so is $\psi=A(z, r) \cos (m \theta)$, as in both cases $\psi_{\theta \theta}=-m^{2} \psi$. Therefore,

$$
\psi_{\mathrm{LG}}^{\text {necklace }}:=\frac{1}{L(z)}\left(\frac{r}{L(z)}\right)^{m} e^{-\frac{1-4 i z}{L^{2}(z)} r^{2}+i(m+1) \zeta(z)} \cos (m \theta), \quad m=1,2, \ldots
$$

are also solutions of (9). Whereas the amplitude of $\psi_{\mathrm{LG}}^{\mathrm{vortex}}$ is radial, that of $\psi_{\mathrm{LG}}^{\text {necklace }}$ has a $|\cos (m \theta)|$ dependence. Hence, $\left|\psi_{\mathrm{LG}}^{\text {necklace }}\right|$ attains its maximum at the $n=2 m$ points

$$
\left(r_{j}, \theta_{j}\right)=\left(r_{m}(z), \frac{j \pi}{m}\right), \quad r_{m}(z):=\arg \max _{r}\left(\left(\frac{r}{L(z)}\right)^{m} e^{-\frac{r^{2}}{L^{2}(z)}}\right), \quad j=1, \ldots, n .
$$

These $n$ peaks are located at equal distances along an expanding circle of radius $r_{m}(z)=$ $r_{m}(0) L(z)$, such that adjacent peaks have a $\pi$ phase difference.

The NLS (1) also admits vortex solutions of the form $\psi=A(z, r) e^{i m \theta}$ (16). In nonlinear propagation, however, it is no longer true that if $\psi=A(z, r) e^{i m \theta}$ is a solution, then so is $\psi=$ $A(z, r) \cos (m \theta)$. Nevertheless, the NLS admits solutions that have a "necklace structure with $n$ pearls":

Lemma 1. Let $\psi(z, r, \theta)$ be a solution of the NLS (1), let $n$ be even, and let

$$
\psi_{0}(r, \theta)=f(r) \cos \left(\frac{n}{2} \theta\right)
$$

Then 
1. $\psi$ is invariant under rotations by $\frac{4 \pi}{n}$, i.e., $\psi(z, r, \theta)=\psi\left(z, r, \theta+\frac{4 \pi}{n}\right)$.

2. $\psi$ is antisymmetric with respect to the $n$ rays $\theta \equiv \Theta_{j}:=\frac{(1+2 j) \pi}{n}$, i.e.,

$$
\psi\left(z, r, \Theta_{j}+\theta\right)=-\psi\left(z, r, \Theta_{j}+\theta\right), \quad j=1, \ldots, n .
$$

Proof. This is a standard result, which follows from the uniqueness of NLS solutions.

The repulsion between adjacent out-of-phase pearls (beams) can be understood as follows. By (11),

$$
\psi\left(z, r, \Theta_{j}\right) \equiv 0, \quad j=1, \ldots, n .
$$

Thus, as the necklace evolves, the electric field remains zero on the rays that separate between adjacent beams (see Figure 1). Hence, the dynamics of the $j$ th pearl is governed by the NLS (3) on the $j$ th sector $D=\left\{\Theta_{j}<\theta<\tilde{\Theta}_{j+1}, 0<r<\infty\right\}$, subject to Dirichlet boundary conditions on the rays $\theta \equiv \Theta_{j}$ and $\theta \equiv \tilde{\Theta}_{j+1}$. Because a Dirichlet boundary condition is reflecting, there is no interaction between adjacent pearls (beams).

Corollary 1. So long that it exists, the solution of the NLS (1) with the necklace initial condition (10) maintains a necklace structure.

If the power of each pearl is sufficiently above the critical power for collapse $P_{\mathrm{cr}}$, then the solution collapses at a finite distance. ${ }^{3}$ In that case, $\psi$ collapses simultaneously at $n$ points. If, however, the pearl power is below $P_{\text {cr }}$, then $\psi$ scatters as $z \rightarrow \infty$. This scattering is slower than for a single pearl, however, since the reflecting boundaries (or equivalently, the repulsion by the adjacent out-of-phase pearls) slow down the pearl expansion. In particular, if the power of each pearl is slightly below $P_{\mathrm{cr}}$, diffraction is almost balanced by the focusing Kerr nonlinearity and the repulsion by the adjacent pearls. Consequently, the pearl expansion is much slower than in the single-pearl case, as is demonstrated numerically in Figure 2.

Let us briefly discuss the stability of necklace beams in free space. By Lemma 1, as long as the initial condition is of the form (10), the necklace structure is preserved. Hence, the necklace structure can only be destroyed by perturbations that are not of the form (10). Such "azimuthal" perturbations break the antisymmetry with respect to the rays $\theta \equiv \Theta_{j}$, and lead to power transfer between adjacent pearls. Generally speaking, numerical simulations suggest that necklace beams in free-space are more stable under random perturbations than vortex beams $(3 ; 2)$.

\subsection{No necklace solitary waves in free space}

Ideally, a stable necklace beam should neither expand nor collapse, i.e., it should be a solitary wave. While the free-space NLS admits necklace-type solutions, see Lemma 1, it does not admit necklace-type solitary waves. This is easy to see for the one-dimensional NLS ${ }^{4}$

$$
i \psi_{z}(z, x)+\psi_{x x}+|\psi|^{2 \sigma} \psi=0, \quad-\infty<x<\infty, \quad z>0 .
$$

\footnotetext{
${ }^{3}$ This follows from the variance identity on a bounded domain.

${ }^{4}$ In one dimension we consider both the subcritical case $\sigma=1$ and the critical case $\sigma=2$.
} 

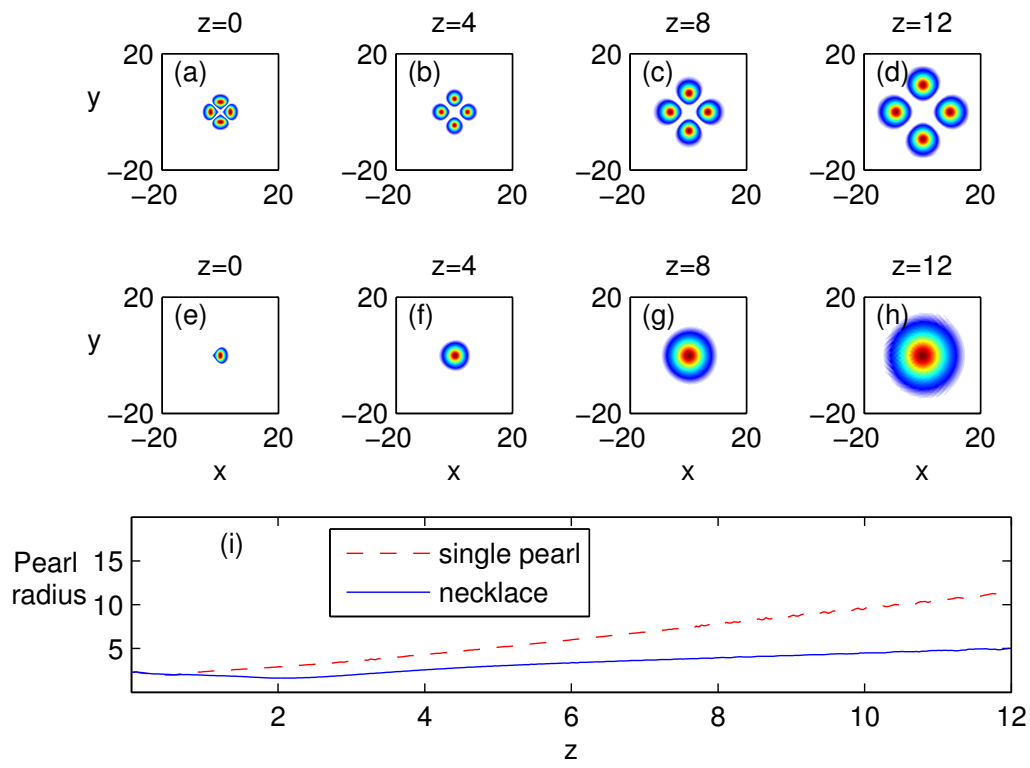

Figure 2: Solution of the NLS (1). (a)-(d): Contour plots of $|\psi|$ at different distances, for $\psi_{0}=\sqrt{2} \operatorname{sech}(r-$ $3.4) \cos (2 \theta)$. (e)-(h): The same when $\psi_{0}$ consists of a single pearl, i.e., $\psi_{0}=\sqrt{2} \operatorname{sech}(r-3.4) \cos (2 \theta)$ for $-\pi / 4 \leq \theta \leq \pi / 4$ and $\psi_{0} \equiv 0$ otherwise. (i): Radius of a single pearl as a function of $z$, for the 4-pearl necklace (solid), and for the single pearl (dashes).

Indeed, the solitary waves of (13) are of the form $\psi_{\mathrm{sw}}=e^{i \mu z} R_{\mu}(x)$, where $R_{\mu}$ is a solution of

$$
R^{\prime \prime}(x)-\mu R+|R|^{2 \sigma} R=0, \quad R( \pm \infty)=0 .
$$

The unique solution of (14) is

$$
R_{\mu, 1 \mathrm{D}}^{(1), \text { free }}(x)=\mu^{\frac{1}{2 \sigma}} R_{1 \mathrm{D}}^{(1), \text { free }}\left(\mu^{\frac{1}{2}} x\right), \quad R_{1 \mathrm{D}}^{(1) \text { free }}(x):=(1+\sigma)^{\frac{1}{2 \sigma}} \operatorname{sech}^{\frac{1}{\sigma}}(\sigma x) .
$$

Since $R_{\mu, 1 \mathrm{D}}^{(1), \text { free }}$ has a single peak, there are no necklace solutions of (14). In two dimensions, we have

Lemma 2. The free-space two-dimensional NLS (2) does not admit necklace-type solitary waves.

Proof. We provide an informal proof. Assume by negation that there is a necklace solution with e.g., $n=4$ pearls. Without loss of generality, their peaks are located at $( \pm a, \pm a)$. By antisymmetry, $R \equiv 0$ on the $x$-axis and $y$-axis. Therefore, there exists a nontrivial, positive, single-peak solution of (4) on the positive quarter-plane $S_{\infty}:=\{0<x, y<\infty\}$, whose peak is at $(a, a)$. By continuity, this solution is the limit of positive, single-peak solutions of (4) on the square $S_{A}:=\{0<x, y<A\}$. By symmetry, however, the peak of these solutions is at $(A / 2, A / 2) .{ }^{5}$ Since $\lim _{A \rightarrow \infty}(A / 2, A / 2) \neq(a, a)$, we reach a contradiction.

\footnotetext{
${ }^{5}$ This statement is further supported by Corollary 2 .
} 


\section{NLS on a bounded domain}

In this section we briefly review the NLS on bounded domain. See (9) and (1, Chapter 16) for further details. Solutions of (3) conserve their power and Hamiltonian, i.e.,

$$
P(\psi) \equiv P\left(\psi_{0}\right), \quad H(\psi) \equiv H\left(\psi_{0}\right)
$$

where

$$
P(\psi):=\int_{D}|\psi|^{2} d x d y, \quad H(\psi):=\int_{D}|\nabla \psi|^{2} d x d y-\frac{1}{2} \int_{D}|\psi|^{4} d x d y .
$$

Recall that the free-space ground-state solitary waves ${ }^{6}$ depend on $\mu$ through the scaling

$$
R_{\mu, 2 \mathrm{D}}^{(1), \text { free }}(\mathbf{x})=\mu^{\frac{1}{2}} R_{2 \mathrm{D}}^{(1), \text { free }}\left(\mu^{\frac{1}{2}} \mathbf{x}\right), \quad R_{2 \mathrm{D}}^{(1), \text { free }}:=R_{\mu=1,2 \mathrm{D}}^{(1), \text { free }}
$$

Consequently, their power is independent of $\mu$, i.e.,

$$
P\left(R_{\mu, 2 \mathrm{D}}^{(1), \text { free }}\right) \equiv P\left(R_{2 \mathrm{D}}^{(1) \text {,ree }}\right)=P_{\mathrm{cr}},
$$

where $P_{\text {cr }}$ is the critical power for collapse. On bounded domains, however, there is no such scaling for $R_{\mu}^{(1)}$. Indeed, numerical simulations and some analytic results show that the power of $R_{\mu}^{(1)}$ is strictly increasing in $\mu$.

The absence of a scaling invariance can be used to give the following variational characterization of the ground-state solitary waves on bounded domains:

Conjecture 1. Let $D$ be a regular bounded domain, and let $\mu_{\text {lin }}$ be the first eigenvalue of (5). Then for all $\mu \in\left(\mu_{\text {lin }}, \infty\right)$,

1. Eq. (4) has minimal-power nontrivial solution, denoted by $R_{\mu}^{(1)}$, which is positive and unique (up to symmetries that leave the domain invariant).

2. $R_{\mu}^{(1)}$ is the unique real minimizer over all $U(x, y) \in H_{0}^{1}(D)$ of

$$
\inf _{\|U\|_{2}^{2}=\left\|R_{\mu}^{(1)}\right\|_{2}^{2}} H(U) .
$$

3. For all $0<P<P_{\text {cr }}$, there exists a unique $\mu_{P} \in\left(\mu_{0}, \infty\right)$ such that $R_{\mu_{P}}^{(1)}$ is the unique real minimizer over all $U(x, y) \in H_{0}^{1}(D)$ of

$$
\inf _{\|U\|_{2}^{2}=P} H(U) .
$$

Parts of Conjecture 1 were proved by Fibich and Merle (9): The existence of a minimizer of (16) was proved for any regular bounded domain, and its uniqueness was proved for $D=B_{1} \cdot{ }^{7}$ Since $H(|u|) \leq H(u)$ for $u \in H_{0}^{1}$, the minimizer can be assumed to be nonnegative.

\footnotetext{
${ }^{6}$ i.e., the positive solutions of (2).

${ }^{7}$ Since then one can use Steiner symmetrization to conclude that the minimizer is radial.
} 
Corollary 2. Assume that Conjecture 1 holds for $D=[-1,1]^{2}$. Then the maximum of $R_{\mu}^{(1)}(x, y)$ is attained at the origin.

Proof. We provide a sketch of a proof. Assume by negation that the maximum of $R_{\mu}^{(1)}$ is not attained at the origin. If we apply Steiner symmetrization to $R_{\mu}^{(1)}(x, y)$ in $x$ and then in $y$, we obtain a function $S_{\mu}(x, y)$ which is symmetric in $x$ and in $y$, and is monotonically decreasing in $|x|$ and in $|y|$. Therefore, its peak is at the origin. Furthermore,

$$
\left\|S_{\mu}\right\|_{2}=\left\|R_{\mu}^{(1)}\right\|_{2}, \quad\left\|S_{\mu}\right\|_{4}=\left\|R_{\mu}^{(1)}\right\|_{4}, \quad\left\|\nabla S_{\mu}\right\|_{2} \leq\left\|\nabla R_{\mu}^{(1)}\right\|_{2}
$$

In fact, we claim that $\left\|\nabla S_{\mu}\right\|_{2}<\left\|\nabla R_{\mu}^{(1)}\right\|_{2}$. Therefore, $S_{\mu} \in H_{0}^{1}(D),\left\|S_{\mu}\right\|_{2}=\left\|R_{\mu}^{(1)}\right\|_{2}$ and $H\left(S_{\mu}\right)<H\left(R_{\mu}^{(1)}\right)$, which is in contradiction to our assumption that $R_{\mu}^{(1)}$ is a minimizer.

Indeed, if $\left\|\nabla S_{\mu}\right\|_{2}=\left\|\nabla R_{\mu}^{(1)}\right\|_{2}$, then $\nabla R_{\mu}^{(1)}$ vanishes on a set of positive measure inside $D$ (see e.g., (17, Thoerem 2.6)). In that case, however, by elliptic regularity, the unique continuation of $R_{\mu}^{(1)}$ from the set of positive measure where $\nabla R_{\mu}^{(1)}$ vanishes to $D$ is $R_{\mu}^{(1)} \equiv 0$.

\section{Necklace solitary waves}

In this section we construct necklace solitary waves on various bounded domains. The numerical method used for computing these solutions is discussed in Section 6.1.

\subsection{Rectangular necklaces}

To construct a necklace solitary wave on a rectangular domain, we first consider a single pearl on a square.

\subsubsection{Single pearl on a square}

Let $D$ be the square $[-1,1]^{2}$. Then equation (4) reads

$$
\begin{gathered}
\Delta R(x, y)-\mu R+|R|^{2} R=0, \quad-1<x, y<1 \\
R(x= \pm 1, y)=R(x, y= \pm 1)=0
\end{gathered}
$$

Since we look for a positive solution, we add the requirement that

$$
R(x, y)>0, \quad-1<x, y<1 .
$$

Following Fibich and Merle (9), the solutions of (17), which we denote by $R_{\mu}^{(1)}$, bifurcate from

$$
Q^{(1)}(x, y):=\cos \left(\frac{\pi x}{2}\right) \cos \left(\frac{\pi y}{2}\right), \quad \mu_{\operatorname{lin}}=-\frac{\pi^{2}}{2},
$$

which is the ground-state eigenpair of (5) with $D=[-1,1]^{2}$.

As $\mu$ increases from $\mu_{\text {lin }}, R_{\mu}^{(1)}$ becomes more localized as its power increases and the nonlinearity becomes more pronounced, see Fig. 3. In particular, as $\mu \rightarrow \infty, R_{\mu}^{(1)}$ "does not feel" the reflecting boundary, and so it approaches the corresponding free-space solitary wave, i.e., $R_{\mu}^{(1)} \sim R_{\mu, 2 \mathrm{D}}^{(1) \text {,free }}$, where $R_{\mu, 2 \mathrm{D}}^{(1), \text { free }}$ is the positive solution of $(2)$. Hence, $\lim _{\mu \rightarrow \infty} P\left(R_{\mu}^{(1)}\right)=$ $P_{\mathrm{cr}}$, where $P_{\mathrm{cr}}=\int\left|R_{2 \mathrm{D}}^{(1), \text { free }}\right|^{2} d x d y$ is the critical power for collapse (18). 


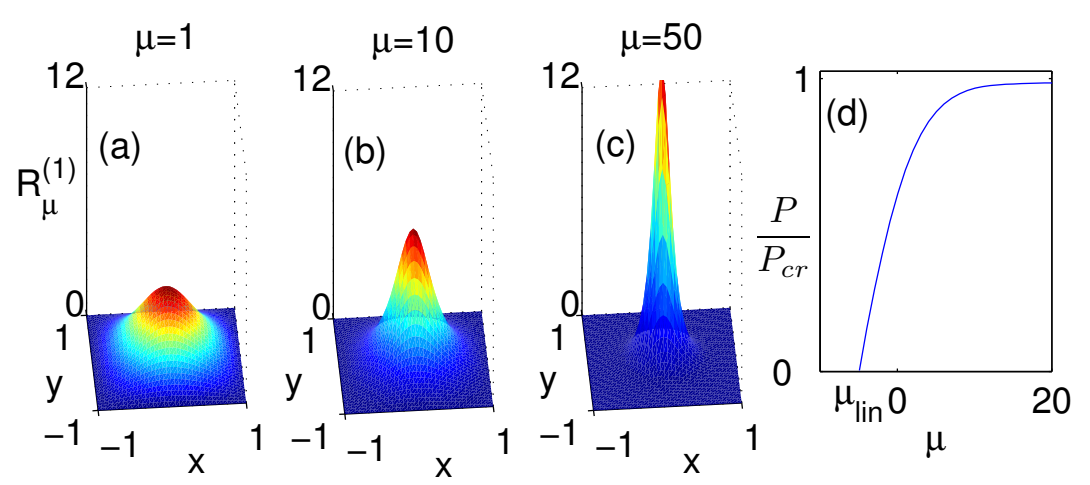

Figure 3: A single pearl on a square, i.e., the solution of (17). (a) $\mu=1$. (b) $\mu=10$. (c) $\mu=50$. (d) The pearl power $P\left(R_{\mu}^{(1)}\right)=\int_{-1}^{1} \int_{-1}^{1}\left|R_{\mu}^{(1)}\right|^{2} d x d y$ as a function of $\mu$. Here, $\mu_{\operatorname{lin}} \approx-5$.

\subsubsection{Rectangular necklace solitary waves}

We can use the single-pearl solution $R_{\mu}^{(1)}$ to construct a rectangular necklace solitary wave with $l \times k$ pearls, by letting adjacent pearls have opposite phases (signs). For example, Fig. 4 shows a rectangular necklace solitary wave with $2 \times 3$ pearls.

Lemma 3. $R_{\mu}^{(l \times k)}$ is a rectangular necklace solution of equation (4) with $l \times k$ pearls.

Proof. Clearly, $R_{\mu}^{(l \times k)}$ is a smooth solution of (4a) inside each "cell", and it satisfies the boundary conditions (4b). To show that $R_{\mu}^{(l \times k)}$ satisfies equation (4a) at the interfaces between pearls, it is enough to show that it is twice continuously differentiable there. Clearly, $R_{\mu}^{(l \times k)}$ is continuous at the interfaces, since the pearls vanish there. Consider, for example, the interface $x \equiv 1$ in Fig. 4(a). Note that if $R_{\mu}^{(1)}(x, y)$ is a solution of (17), then so is $R_{\mu}^{(1)}(-x, y)$. Therefore, $R_{\mu}^{(1)}(-x, y)=R_{\mu}^{(1)}(x, y)$, and so

$$
-\frac{\partial}{\partial x} R_{\mu}^{(l \times k)}(x=-1+, y)=\frac{\partial}{\partial x} R_{\mu}^{(l \times k)}(x=1-, y) .
$$

In addition, by construction of $R_{\mu}^{(l \times k)}$, we have that $R_{\mu}^{(l \times k)}(x, y)=-R_{\mu}^{(l \times k)}(x-2, y)$. Hence,

$$
\frac{\partial}{\partial x} R_{\mu}^{(l \times k)}(x=1+, y)=-\frac{\partial}{\partial x} R_{\mu}^{(l \times k)}(x=-1+, y) .
$$

Therefore, by (19), $\frac{\partial}{\partial x} R_{\mu}^{(l \times k)}$ is continuous at $x \equiv 1$. Since $R_{\mu}^{(l \times k)} \equiv 0$ on the interface $x \equiv 1$, then $\frac{\partial}{\partial y} R_{\mu}^{(l \times k)} \equiv 0, \frac{\partial^{2}}{\partial x \partial y} R_{\mu}^{(l \times k)} \equiv 0$, and $\frac{\partial^{2}}{\partial y^{2}} R_{\mu}^{(l \times k)} \equiv 0$ there. Therefore, $\frac{\partial^{2}}{\partial x^{2}} R_{\mu}^{(l \times k)}=(\mu-$ $\left.\left|R_{\mu}^{(l \times k)}\right|^{2}\right) R_{\mu}^{(l \times k)}-\frac{\partial^{2}}{\partial y^{2}} R_{\mu}^{(l \times k)} \equiv 0$ there. Hence, $\frac{\partial}{\partial y} R_{\mu}^{(l \times k)}, \frac{\partial^{2}}{\partial x \partial y} R_{\mu}^{(l \times k)}, \frac{\partial^{2}}{\partial y^{2}} R_{\mu}^{(l \times k)}$ and $\frac{\partial^{2}}{\partial x^{2}} R_{\mu}^{(l \times k)}$ are also continuous at $x \equiv 1$. 
Remark. Note that $R_{\mu}^{(l \times k)}$ is identically zero along the lines separating adjacent pearls, and is antisymmetric with respect to these lines.

(a)

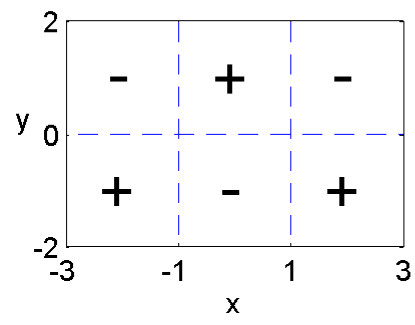

(b)

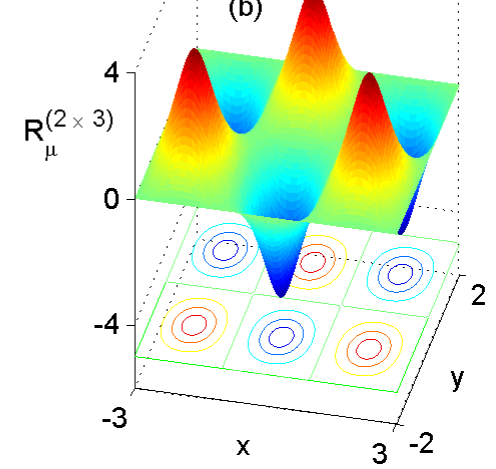

Figure 4: (a) Construction of a rectangular necklace solitary wave with $2 \times 3$ pearls. The ' $\pm^{\prime}$ symbols correspond to $\pm R_{\mu}^{(1)}$. Note that $R_{\mu}^{(2 \times 3)}$ is identically zero along the dashed lines, and is antisymmetric with respect to these lines. (b) The rectangular necklace solitary wave $R_{\mu=1}^{(2 \times 3)}$.

Remark. The single-pearl solution can also be used to construct necklace solitary waves on non-rectangular domains, see e.g., Figure 5.
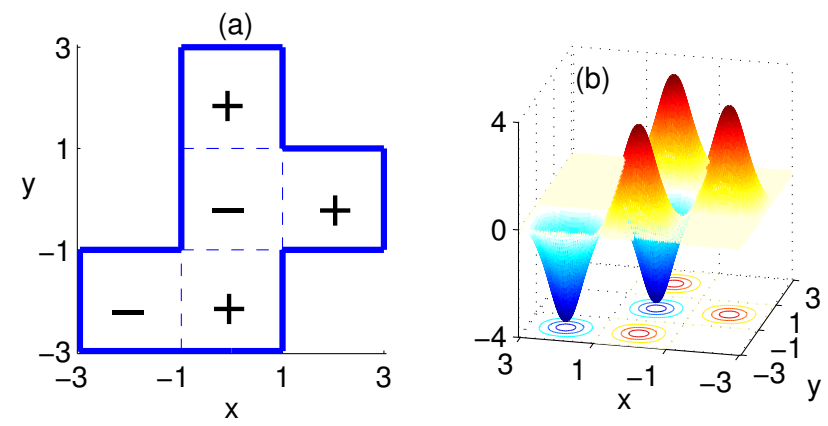

Figure 5: Same as Fig. 4 for a non-rectangular domain with 5 pearls.

\subsection{Circular necklaces}

To construct a necklace solitary wave on a circular domain, we repeat the procedure for a rectangular domain (Section 4.1). Thus, we first compute a single pearl solution on a sector of a circle, and then use it to construct a circular necklace solitary wave. 


\subsubsection{Single pearl on a sector of a circle}

Let $D=\left\{r \in[0,1], \theta \in\left[0, \frac{2 \pi}{n}\right]\right\}$ be the sector of the unit circle. Then equation (4) reads

$$
\begin{gathered}
R_{r r}(r, \theta)+\frac{1}{r} R_{r}+\frac{1}{r^{2}} R_{\theta \theta}-\mu R+|R|^{2} R=0, \quad 0<r<1, \quad 0<\theta<\frac{2 \pi}{n}, \\
R(r=1, \theta)=R(r, \theta=0)=R\left(r, \theta=\frac{2 \pi}{n}\right)=0 .
\end{gathered}
$$

Since we look for a positive solution, we add the requirement that

$$
R(r, \theta)>0, \quad 0<r<1, \quad 0<\theta<\frac{2 \pi}{n} .
$$

Solutions of (20) bifurcate from the positive eigenfunction of (5) with $D=\{r \in[0,1], \theta \in$ $\left.\left[0, \frac{2 \pi}{n}\right]\right\}$, which is given by

$$
Q^{(1)}:=J_{\frac{n}{2}}\left(k_{\frac{n}{2}} r\right) \sin \left(\frac{n}{2} \theta\right), \quad \mu_{\text {lin }}=-k_{\frac{n}{2}}^{2},
$$

where $J_{\frac{n}{2}}$ is the Bessel function of order $\frac{n}{2}$ of the first kind, and $k_{\frac{n}{2}}$ is the first positive root of $J_{\frac{n}{2}}$. As $\mu$ increases from $\mu_{\text {lin }}, R_{\mu}^{(1)}$ becomes more localized as its power increases, see Fig. 6. In particular, $R_{\mu}^{(1)} \sim R_{\mu, 2 \mathrm{D}}^{(1) \text { free }}$ as $\mu \rightarrow \infty$. Consequently, $\lim _{\mu \rightarrow \infty} P\left(R_{\mu}^{(1)}\right)=P_{\mathrm{cr}}$.
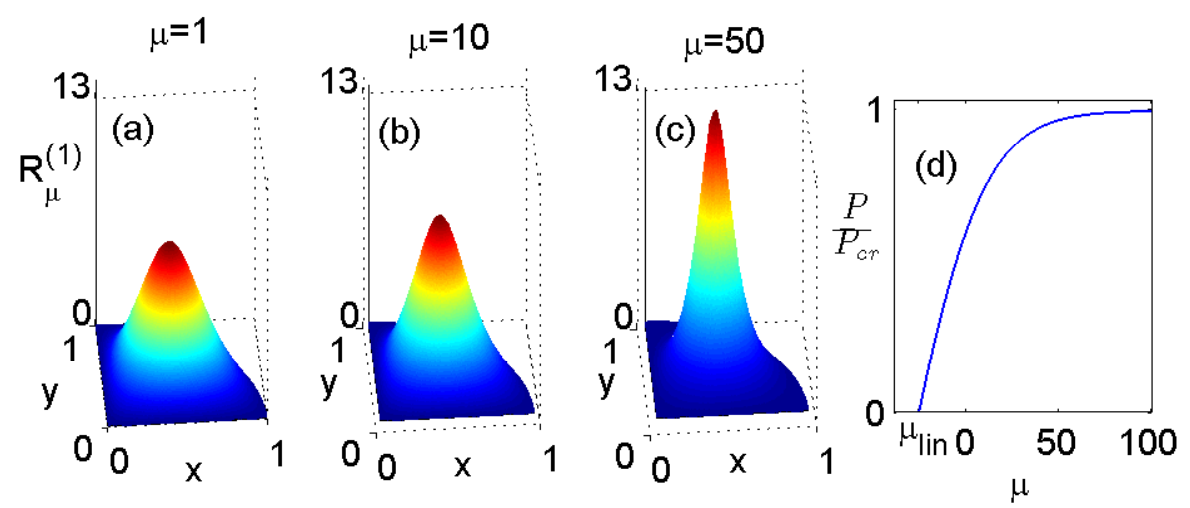

Figure 6: A single pearl on a quarter of the unit circle, i.e., the ground-state solution of (20) with $n=4$. (a) $\mu=1$. (b) $\mu=10$. (c) $\mu=50$. (d) The pearl power $P\left(R_{\mu}^{(1)}\right)=\int_{0}^{\frac{\pi}{2}} \int_{0}^{1} r\left|R_{\mu}^{(1)}\right|^{2} d r d \theta$ as a function of $\mu$. Here, $\mu_{\text {lin }} \approx-26.4$.

\subsubsection{Circular necklace solitary waves}

When $n$ is even, we can use the single-pearl solution $R_{\mu}^{(1)}$ to construct a circular necklace solitary wave with $n$ pearls, by letting neighboring pearls have opposite signs. For example, 
Fig. 7 shows a circular necklace solitary wave with 4 pearls. Note that $R_{\mu}^{(n)}$ is identically zero along the rays that are half way through between adjacent pearls, and is antisymmetric with respect to these rays.

Lemma 4. Let $n$ be even. Then $R_{\mu}^{(n)}$ is a circular necklace solution of equation (4) with $n$ pearls.

Proof. The proof is the same as that of Lemma 3.
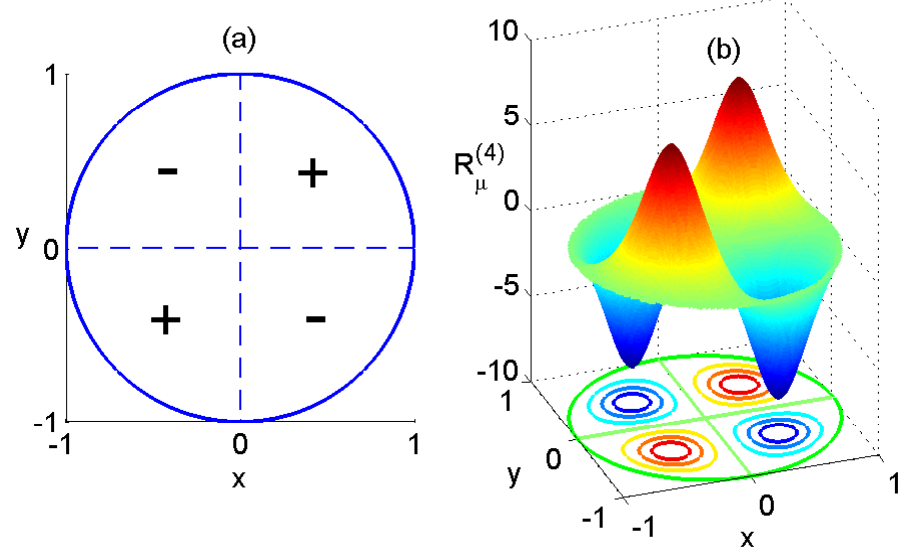

Figure 7: a) Construction of the circular necklace solitary wave with 4 pearls. The symbols ' $\pm^{\prime}$ correspond to $\pm R_{\mu}^{(1)}$. Note that $R_{\mu}^{(4)}$ is identically zero along the dashed lines, and is antisymmetric with respect to these lines. (b) The circular necklace solitary wave $R_{\mu=1}^{(4)}$.

\subsection{Annular necklaces}

To construct a necklace solitary wave on an annular domain, we first consider a single pearl on a sector of an annulus, and then use it to construct necklace solutions on the whole annular domain.

\subsubsection{Single pearl on a sector of an annulus}

Let $D=\left\{r \in\left[r_{\min }, r_{\max }\right], \theta \in\left[0, \frac{2 \pi}{n}\right]\right\}$ be the sector of the annulus. Then equation (4) reads

$$
\begin{gathered}
R_{r r}(r, \theta)+\frac{1}{r} R_{r}+\frac{1}{r^{2}} R_{\theta \theta}-\mu R+|R|^{2} R=0, \quad r_{\min }<r<r_{\max }, \quad 0<\theta<\frac{2 \pi}{n}, \\
R\left(r_{\min }, \theta\right)=R\left(r_{\max }, \theta\right)=R(r, \theta=0)=R\left(r, \theta=\frac{2 \pi}{n}\right)=0 .
\end{gathered}
$$

Since we look for a positive solution, we add the requirement that

$$
R(r, \theta)>0, \quad r_{\min }<r<r_{\max }, \quad 0<\theta<\frac{2 \pi}{n} .
$$


Solutions of (22) bifurcate from the ground-state eigenfunction of (5) with $D=\{r \in$ $\left.\left[r_{\min }, r_{\max }\right], \theta \in\left[0, \frac{2 \pi}{n}\right]\right\}$, which is given by

$$
Q^{(1)}=\left[J_{\frac{n}{2}}\left(k_{\frac{n}{2}} r\right)-\frac{J_{\frac{n}{2}}\left(k_{\frac{n}{2}} r_{\min }\right)}{Y_{\frac{n}{2}}\left(k_{\frac{n}{2}} r_{\min }\right)} Y_{\frac{n}{2}}\left(k_{\frac{n}{2}} r\right)\right] \sin \left(\frac{n \theta}{2}\right), \quad \mu_{\operatorname{lin}}=-k_{\frac{n}{2}}^{2},
$$

where $J_{\frac{n}{2}}$ and $Y_{\frac{n}{2}}$ are Bessel functions of order $\frac{n}{2}$ of the first and second kind, respectively, and $k_{\frac{n}{2}}$ is the smallest positive root of

$$
J_{\frac{n}{2}}\left(k_{\frac{n}{2}} r_{\max }\right) Y_{\frac{n}{2}}\left(k_{\frac{n}{2}} r_{\min }\right)-Y_{\frac{n}{2}}\left(k_{\frac{n}{2}} r_{\max }\right) J_{\frac{n}{2}}\left(k_{\frac{n}{2}} r_{\min }\right)=0 .
$$

As $\mu$ increases from $\mu_{\text {lin }}, R_{\mu}^{(1)}$ becomes more localized as its power increases, see Fig. 8. In particular, $R_{\mu}^{(1)} \sim R_{\mu, 2 \mathrm{D}}^{(1) \text {,free }}$ as $\mu \rightarrow \infty$. Consequently, $\lim _{\mu \rightarrow \infty} P\left(R_{\mu}^{(1)}\right)=P_{\mathrm{cr}}$.

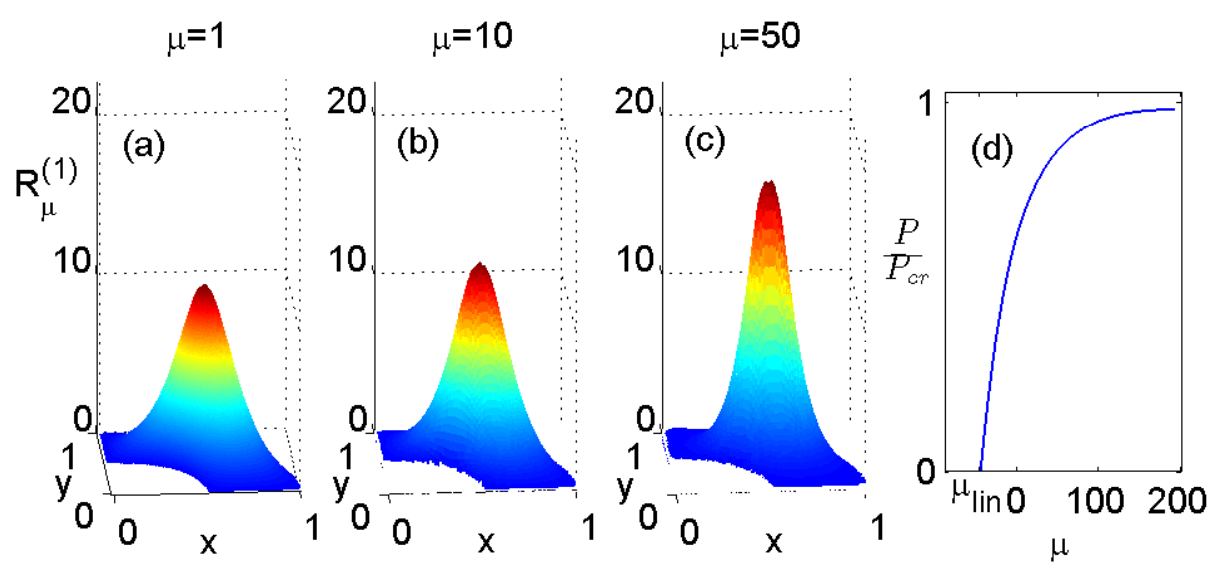

Figure 8: A single pearl on a quarter annulus, i.e., the solution of (22) with $n=4$. a) $\mu=1$. (b) $\mu=10$. (c) $\mu=50$. (d) The pearl power $P\left(R_{\mu}^{(1)}\right)=\int_{0}^{\frac{2 \pi}{n}} d \theta \int_{r_{\min }}^{r_{\max }} r\left|R_{\mu}^{(1)}\right|^{2} d r$ as a function of $\mu$. Here $r_{\min }=0.5$, $r_{\max }=1$, and $\mu_{\text {lin }} \approx-45.5$.

\subsubsection{Annular necklace solitary waves}

We can use $R_{\mu}^{(1)}$ as a "building block" to construct an annular necklace solitary wave with $n$ pearls, by letting neighboring pearls have opposite signs. For example, Fig. 9 shows an annular necklace solitary wave with 4 pearls.

Lemma 5. Let $n$ be even. Then $R_{\mu}^{(n)}$ is an annular necklace solution of equation (4) with $n$ pearls.

Proof. The proof is the same as that of Lemma 3. 

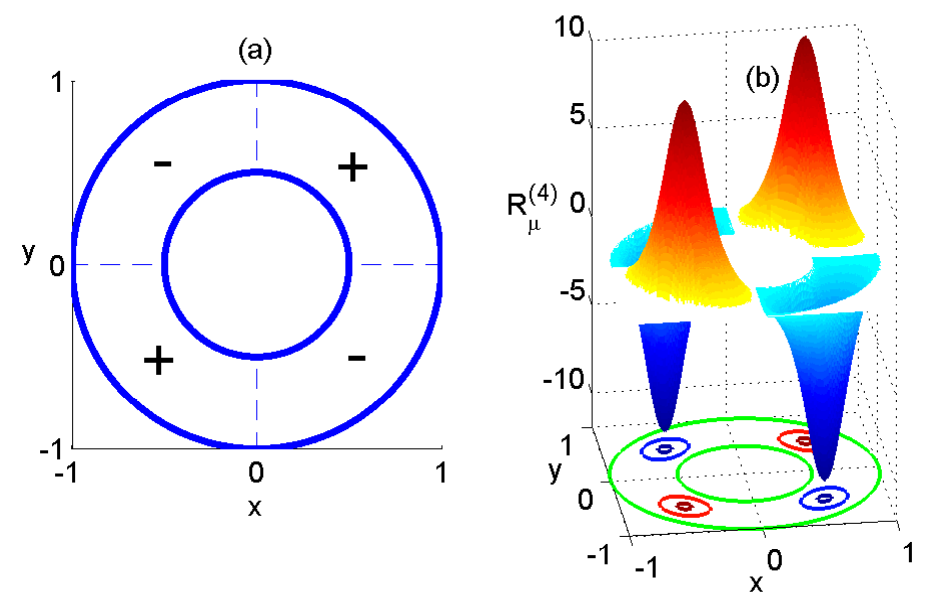

Figure 9: (a) Construction of an annular necklace solitary wave with 4 pearls. The symbols ' $\pm^{\prime}$ correspond to $\pm R_{\mu}^{(1)}$. Note that $R_{\mu}^{(4)}$ is identically zero along the dashed lines, and is antisymmetric with respect to these lines. (b) The annular necklace solitary wave $R_{\mu=1}^{(4)}$. Here $r_{\min }=0.5$ and $r_{\max }=1$.

\subsubsection{Annular ground-state solitary waves (symmetry breaking)}

We can look for positive radial solitary waves $\psi_{\mathrm{sw}}=e^{i \mu z} R_{\mu}^{\text {ring }}(r)$ of the NLS (3) on the annulus. The profile of these ring-type solitary waves satisfies

$$
\begin{gathered}
R_{r r}(r)+\frac{1}{r} R_{r}-\mu R+|R|^{2} R=0, \quad r_{\text {min }}<r<r_{\text {max }}, \\
R\left(r_{\text {min }}\right)=R\left(r_{\max }\right)=0, \\
R(r)>0, \quad r_{\text {min }}<r<r_{\text {max }} .
\end{gathered}
$$

Because of the hole and the requirement of radial symmetry, these solitary waves cannot approach the free-space two-dimensional ground-state $R_{\mu, 2 \mathrm{D}}^{(1) \text {,free }}$ as $\mu \rightarrow \infty$. Rather,

$$
R_{\mu}^{\text {ring }} \sim \sqrt{\mu} R_{1 \mathrm{D}}^{(1), \text { free }}\left(\sqrt{\mu}\left(r-r_{\mathrm{M}, \mu}\right)\right), \quad \mu \rightarrow \infty,
$$

where $R_{1 \mathrm{D}}^{(1) \text { free }}$ is the positive solution of (14) and $r_{\mathrm{M}, \mu}:=\arg \max R_{\mu}^{\mathrm{ring}}(r)$. Hence,

$$
P\left(R_{\mu}^{\text {ring }}\right)=2 \pi \int_{r_{\min }}^{r_{\max }}\left|R_{\mu}^{\text {ring }}\right|^{2} r d r \sim 2 \pi \int_{r_{\min }}^{r_{\max }}\left|\sqrt{\mu} R_{1 \mathrm{D}}^{(1), \text { free }}\left(\sqrt{\mu}\left(r-r_{\mathrm{M}, \mu}\right)\right)\right|^{2} r d r \sim c \sqrt{\mu}
$$

where $c=2 \pi r_{\mathrm{M}, \mu} \int_{-\infty}^{\infty}\left|R_{1 \mathrm{D}}^{(1) \text { free }}(x)\right|^{2} d x$. Consequently, $\lim _{\mu \rightarrow \infty} P\left(R_{\mu}^{\text {ring }}\right)=\infty$.

In Figure 10 we compute the ground-state solutions of (4) on the annulus $\{0.5<|\mathbf{x}|<1\}$ using the non-spectral renormalization method (Section 6.1) without imposing radial symmetry. These positive solutions are radial for $\mu_{\operatorname{lin}}<\mu<\mu_{\mathrm{c}},{ }^{8}$ where $\mu_{\operatorname{lin}} \approx-39$ and $\mu_{\mathrm{c}} \approx-38.2$.

\footnotetext{
8i.e., $R_{\mu}^{(1)}=R_{\mu}^{\text {ring }}$ for $\mu_{\text {lin }}<\mu<\mu_{\mathrm{c}}$.
} 
For $\mu_{\mathrm{c}}<\mu<\infty$, they become non-radial. In particular, $R_{\mu}^{(1)} \sim \sqrt{\mu} R_{1 \mathrm{D}}^{(2) \text {,free }}\left(\sqrt{\mu}\left(\mathbf{x}-\mathbf{x}_{\mathrm{M}, \mu}\right)\right)$ as $\mu \rightarrow \infty .{ }^{9}$ Consequently, $\lim _{\mu \rightarrow \infty} P\left(R_{\mu}^{(1)}\right)=P_{\mathrm{cr}}$.

The symmetry-breaking at $\mu_{\mathrm{c}}$ is related to the variational characterization of the groundstate (see Conjecture 1). Indeed, by (25), $H\left(R_{\mu}^{\text {ring }}\right) \sim \mu^{3 / 2}$ as $\mu \rightarrow \infty$. Therefore, high-power solutions of (4) on $D=\left\{r_{\min }<|\mathbf{x}|<r_{\max }\right\}$ can achieve a smaller Hamiltonian by "adopting" a non-radial profile.

Note that for $\mu_{\mathrm{c}}<\mu<\infty$, equation (4) on the annulus admits both a radial solution and a non-radial one. To the best of our knowledge, this is the first example that equation (4) admits two different positive solutions, and that a positive solution of (4) is not a ground state. As noted above, such results were observed for the inhomogeneous NLS (12).
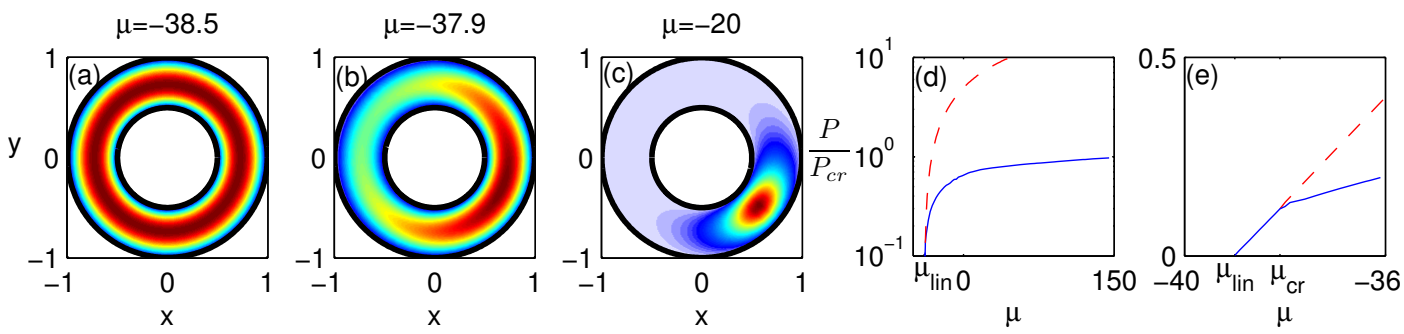

Figure 10: Ground-state solutions of (4) on the annulus $\left\{r_{\min }<|\mathbf{x}|<r_{\max }\right\}$, where $r_{\min }=0.5$ and $r_{\max }=1$. Here $\mu_{\operatorname{lin}}=-38.2$. (a) $\mu=-38.5$. (b) $\mu=-37.9$. (c) $\mu=-20$. (d) $P\left(R_{\mu}^{(1)}\right)$ as a function of $\mu$ (solid blue line). The dashed red line is $P\left(R_{\mu}^{\text {ring }}\right)$. e) Zoom in near the bifurcation at $\mu_{c}$.

\subsection{One-dimensional necklaces}

It is instructive to consider "necklace solutions" in one dimension. While less of physical interest, they allow for simpler analysis and simulations than their two-dimensional counterparts.

Let $\psi(z, x)$ be the solution of the one-dimensional NLS on $D=\left[x_{\mathrm{L}}, x_{\mathrm{R}}\right]$

$$
i \psi_{z}(z, x)+\psi_{x x}+|\psi|^{2 \sigma} \psi=0, \quad x_{\mathrm{L}}<x<x_{\mathrm{R}}, \quad z>0,
$$

with the Dirichlet condition

$$
\psi\left(z, x_{\mathrm{L}}\right)=\psi\left(z, x_{\mathrm{R}}\right)=0, \quad z \geq 0
$$

Eq. (26) admits solitary wave solutions $\psi_{\mathrm{sw}}=e^{i \mu z} R_{\mu}(x)$, where $R_{\mu}$ is a solution of

$$
R_{x x}(x)-\mu R+|R|^{2 \sigma} R=0, \quad x_{\mathrm{L}}<x<x_{\mathrm{R}}, \quad R\left(x_{\mathrm{L}}\right)=R\left(x_{\mathrm{R}}\right)=0 .
$$

\footnotetext{
${ }^{9}$ Non-radial ground states on the annulus are only determined up to a rotation about the origin. Therefore, $\mathbf{x}_{\mathrm{M}, \mu}=r_{\mathrm{M}, \mu}(\cos \theta, \sin \theta)$, where $\theta$ is arbitrary.
} 
Let $D$ be the interval $[-1,1]$. Then equation $(27)$ reads

$$
R_{x x}(x)-\mu R+|R|^{2 \sigma} R=0, \quad-1<x<1, \quad R( \pm 1)=0 .
$$

The ground-state solitary waves $R_{\mu}^{(1)}(x)$ of (28) bifurcate from the ground-state linear mode

$$
Q^{(1)}(x):=\cos \left(\frac{\pi x}{2}\right), \quad \mu_{\mathrm{lin}}=-\frac{\pi^{2}}{4}
$$

As $\mu$ increases from $\mu_{\text {lin }}, R_{\mu}^{(1)}$ becomes more localized as its power increases and the nonlinearity becomes more pronounced, see Figure 11. In particular, $R_{\mu}^{(1)} \sim R_{\mu, 1 \mathrm{D}}^{(1) \text {,free }}$ as $\mu \rightarrow \infty$, where $R_{\mu, 1 \mathrm{D}}^{(1) \text {,free }}$ is the positive solution of (14).
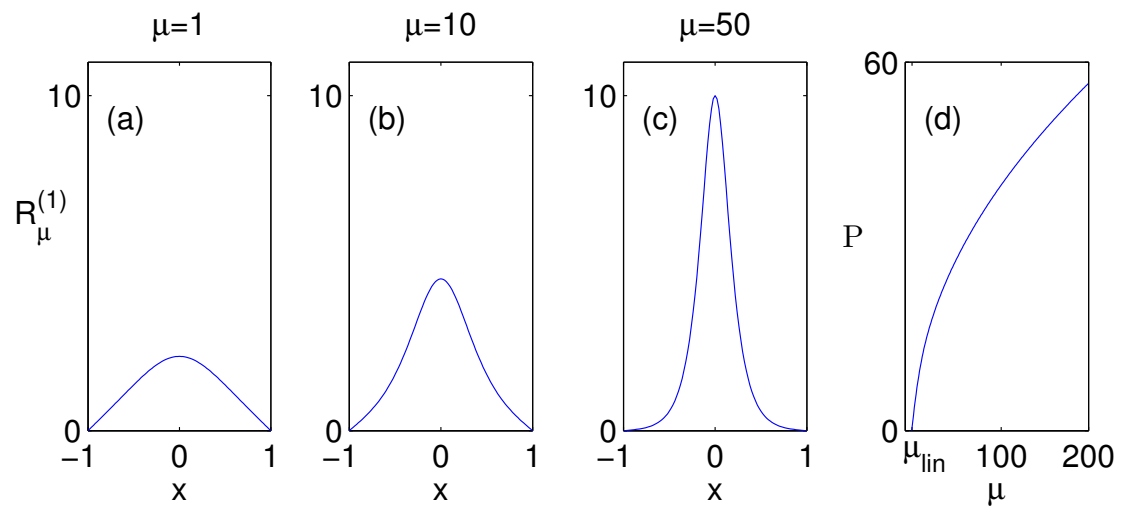

Figure 11: A single pearl on an interval, i.e., the ground-state solution of (28) with $\sigma=1$. (a) $\mu=1$. (b) $\mu=10$. (c) $\mu=50$. (d) The pearl power $P\left(R_{\mu}^{(1)}\right)=\int_{-1}^{1}\left|R_{\mu}^{(1)}\right|^{2} d x$ as a function of $\mu$. Here, $\mu_{\text {lin }} \approx-2.5$.

We can use $R_{\mu}^{(1)}$ to construct a one-dimensional necklace solitary wave with $n$ pearls on the interval $[-n, n]$, by letting adjacent pearls have opposite phases (signs). For example, Fig. 12 shows a one-dimensional necklace solitary wave with 3 pearls.

\section{Stability}

The appropriate notion for stability of NLS solitary waves is that of orbital stability. On a bounded domain, orbital stability refers to stability up to phase shifts:

Definition 3 (orbital stability). Let $\psi_{\mathrm{sw}}=e^{i \mu z} R_{\mu}$ be a solitary wave solution of (3). We say that $\psi_{\mathrm{sw}}$ is orbitally stable, if for any $\epsilon>0$, there exists $\delta>0$, such that if $\left\|\psi_{0}-R_{\mu}\right\|_{H_{0}^{1}(D)}<\delta$, and if $\psi$ is the solution of (3) with the initial condition $\psi_{0}$, then

$$
\inf _{\theta(z) \in \mathbb{R}}\left\|\psi(z, \mathbf{x})-e^{i \theta(z)} \psi_{\mathrm{sw}}(z, \mathbf{x})\right\|_{H_{0}^{1}(D)}<\epsilon, \quad 0 \leq z<\infty
$$



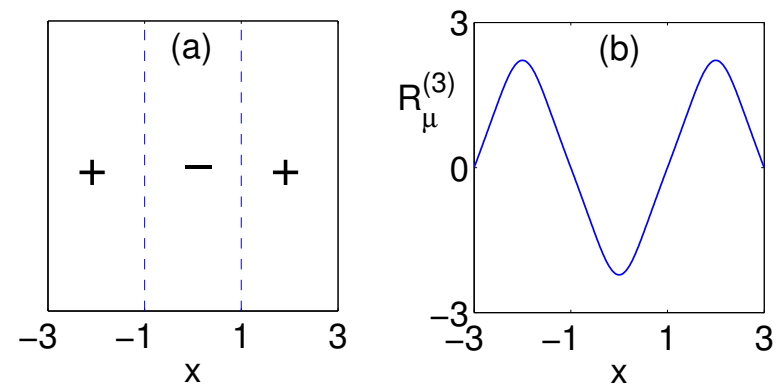

Figure 12: a) Construction of a one-dimensional necklace solitary wave with 3 pearls. The symbols ' $\pm^{\prime}$ correspond to $\pm R_{\mu}^{(1)}$. Note that $R_{\mu}^{(3)}$ is identically zero at $x= \pm 1$, and is antisymmetric with respect to these points. (b) The one-dimensional necklace solitary wave $R_{\mu=1}^{(3)}$.

\subsection{Stability of single-pearl (ground-state) solitary waves}

We recall that in free space, the ground-state solitary waves of the critical NLS (1) are unstable. A reflecting boundary, however, has a stabilizing effect. Indeed, since the VK condition $\frac{d}{d \mu} P\left(R_{\mu}\right)>0$ holds for the ground state solitary waves on $B_{1}$, these solitary waves are orbitally stable for $\mu_{\text {lin }}<\mu<\infty\left(0<P<P_{\text {cr }}\right)$. See $(9 ; 10)$ for more details.

\subsubsection{Convex domains}

Our numerical simulations suggest that the VK condition holds for the ground state solutions of (4) on a square, on a sector of a circle, and on a sector of an annulus (see Fig. 3(d), Fig. 6(d), and Fig. 8(d), respectively). In addition, solving the NLS (3) with an initial condition which is a perturbed single pearl suggests that single-pearl solitary waves are stable, see e.g., Fig. 13. Therefore, based on this numerical evidence, we formulate

Conjecture 2. All single-pearl (positive, ground state) solitary waves $\psi_{\mathrm{sw}}^{(1)}=e^{i \mu z} R_{\mu}^{(1)}(x, y)$ of the NLS (3) on a convex bounded domain are orbitally stable.

\subsubsection{Annular domain}

In the case of an annular domain, there are two types of positive solitary waves: Radial ring-type solutions $\psi_{\mathrm{sw}}^{\mathrm{ring}}=e^{i \mu z} R_{\mu}^{\mathrm{ring}}(r)$, which exist for $\mu_{\mathrm{lin}}<\mu<\infty$, and nonradial singlepeak solutions $\psi_{\mathrm{sw}}^{(1)}=e^{i \mu z} R_{\mu}^{(1)}(r, \theta)$, which exist for $\mu_{\mathrm{c}}<\mu<\infty$, see Section 4.3.3. The ground states solutions (i.e., the minimizers of the Hamiltonian, see Conjecture 1) are radial for $\mu_{\text {lin }}<\mu<\mu_{\mathrm{c}}$ and non-radial for $\mu_{\mathrm{c}}<\mu<\infty$.

To test numerically for stability, we solve the NLS with an initial condition which is the solitary wave profile, multiplied by 1.05 in the first quadrant. Thus, $\psi_{0}=R_{\mu}^{\mathrm{ring}}(r) H(\theta)$ or $\psi_{0}=R_{\mu}^{(1)}(r) H(\theta)$, where

$$
H(\theta)=\left\{\begin{array}{cl}
1.05, & \text { for } 0<\theta<\pi / 2 \\
1, & \text { for } \pi / 2<\theta<2 \pi
\end{array}\right.
$$




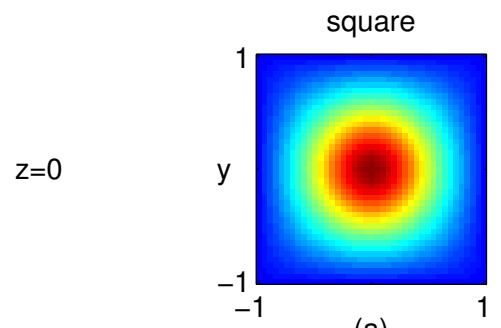

(a)

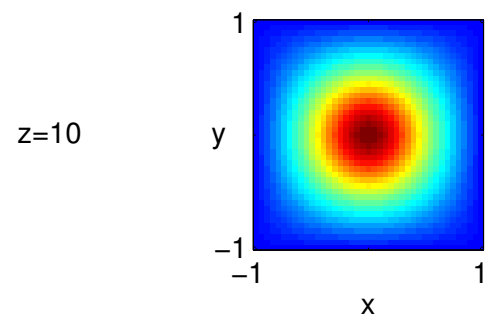

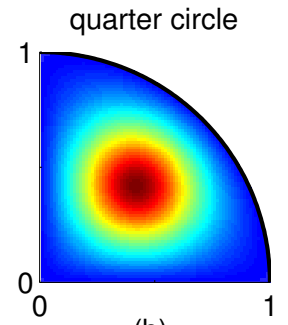

(b)

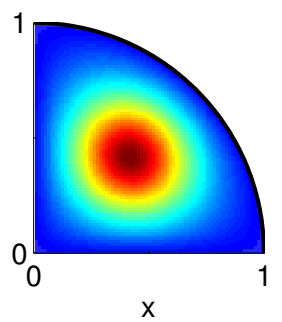

quarter annulus

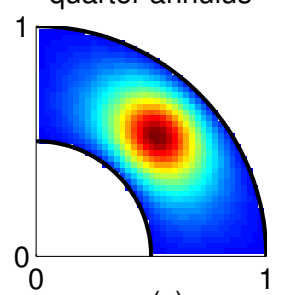

(c)

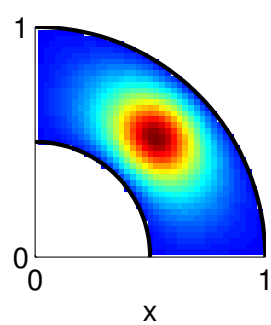

Figure 13: Contour plots of $|\psi|$ at $z=0$ (top) and $z=10$ (bottom). Here $\psi$ is the solution of the NLS (3) on a domain $D$, with the perturbed single-pearl initial condition $\psi_{0}=1.05 R_{\mu=1}^{(1)}(x, y)$. (a) $D$ is the square $[-1,1]^{2}$. (b) $D$ is the quarter circle $\left\{0 \leq r \leq 1,0 \leq \theta \leq \frac{\pi}{2}\right\}$. (c) $D$ is the quarter annulus $\left\{\frac{1}{2} \leq r \leq 1,0 \leq \theta \leq \frac{\pi}{2}\right\}$.

Numerical simulations (Figure 14 top and bottom raws) show that the ground state solitary waves $\psi_{\mathrm{sw}}^{(1)}$ are stable both below and above the symmetry-breaking point $\mu_{\mathrm{cr}}$ (i.e., for $\mu_{\text {lin }}<$ $\mu<\infty)$. The ring-type solitary waves $\psi_{\mathrm{sw}}^{\mathrm{ring}}$, however, are unstable for $\mu_{\mathrm{cr}}<\mu<\infty$, see Figure 14 (middle raw). Thus, as expected, the ground-state solutions are stable, but the excited ones are not. ${ }^{10}, 11$

\subsection{Stability of necklace solitary waves}

Since each pearl satisfies the VK condition (Section 5.1), so does the necklace, see (7). This does not imply that the necklace is stable, however, since the VK condition implies stability only for ground states. Therefore, to check for necklace stability, we have to "go back" to the original eigenvalue problem from which the VK condition was derived (19).

Let

$$
\psi=e^{i \mu t}(R(\mathbf{x})+\epsilon h(z, \mathbf{x})), \quad h(z, \mathbf{x})=e^{\Omega z}(h(z, \mathbf{x})+i v(\mathbf{x})) .
$$

Then the linearized equation for $h$ reads

$$
\left(\begin{array}{cc}
0 & L_{+} \\
-L_{-} & 0
\end{array}\right)\left(\begin{array}{l}
v \\
u
\end{array}\right)=\Omega\left(\begin{array}{l}
v \\
u
\end{array}\right)
$$

where $\Omega \in \mathbb{C}$ is the eigenvalue, $\left(\begin{array}{c}v \\ u\end{array}\right)=\left(\begin{array}{l}v(x, y) \\ u(x, y)\end{array}\right)$ is the eigenfunction, and

$$
L_{+}:=\Delta-\mu+3\left|R_{\mu}\right|^{2}, \quad L_{-}:=\Delta-\mu+\left|R_{\mu}\right|^{2} .
$$

\footnotetext{
${ }^{10} \mathrm{~A}$ similar stability pattern, below and above the symmetry-breaking point, was observed for the inhomogeneous NLS (12).

${ }^{11}$ Intuitively, the high-power ring-type solitary waves inherit the azimuthal instability of the free-space ring-type solutions.
} 

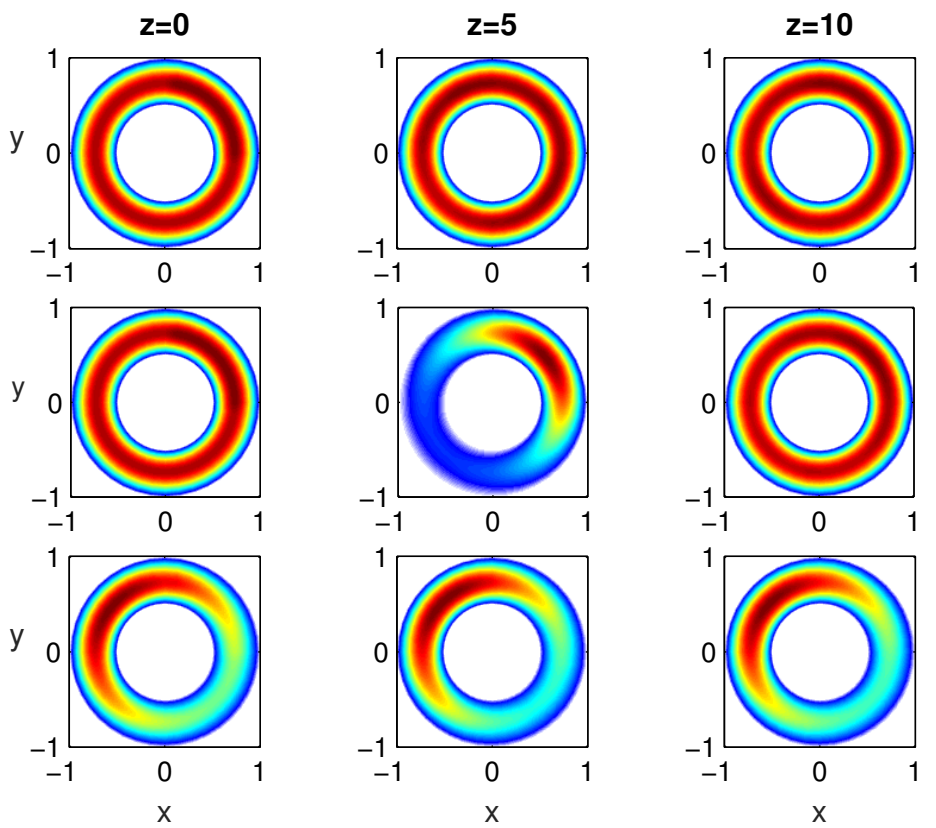

Figure 14: Solution of the NLS (3) on the annular domain $D=\{0.5 \leq|\mathbf{x}| \leq 1\}$. Top row: $\mu=\mu_{\mathrm{cr}}-0.3$ and $\psi_{0}=R_{\mu}^{\text {ring }}(r) H(\theta)$, where $H(\theta)$ is given by (30). Middle row: $\mu=\mu_{\mathrm{cr}}+0.3$ and $\psi_{0}=R_{\mu}^{\text {ring }}(r) H(\theta)$. Bottom row: $\mu=\mu_{\mathrm{cr}}+0.3$ and $\psi_{0}=R_{\mu}^{(1)}(r, \theta) H(\theta)$. Here $\mu_{\mathrm{cr}} \approx-38.2$.

Note that if $\Omega$ is an eigenvalue with eigenvector $\left(\begin{array}{l}v \\ u\end{array}\right)$, then $-\Omega$ is an eigenvalue with eigenvector $\left(\begin{array}{c}-v \\ u\end{array}\right)$, and $\Omega^{*}$ is an eigenvalue with eigenvector $\left(\begin{array}{c}v^{*} \\ u^{*}\end{array}\right)$. Therefore, the genuinely-complex eigenvalues of (31) always come in quadruples. Consequently, without loss of generality, we focus from now on eigenvalues with nonnegative real and imaginary parts.

Necklace solitary waves are stable under perturbations that preserve their symmetries (or, more precisely, their anti-symmetries with respect to the interfaces between adjacent pearls):

Lemma 6. Let $\psi_{\mathrm{sw}}^{(n)}$ be a necklace solitary wave with $n$ pearls. If the single-pearl solitary wave is stable, then $\psi_{\mathrm{sw}}^{(n)}$ is stable under perturbations that preserve the symmetries of $\psi_{\mathrm{sw}}^{(n)}$.

Proof. As in free space, see Lemma 1 and (12), the solution remains zero on the interfaces between pearls. Therefore, there is no interaction between pearls. Hence, if the single pearl is stable, then so is the necklace.

Remark. As $\mu \rightarrow \infty$, the single pearl has the asymptotic scaling

$$
R_{\mu}^{(1)} \sim R_{\mu, 2 \mathrm{D}}^{(1), \text { free }}(r)=\mu^{\frac{1}{2}} R_{2 \mathrm{D}}^{(1), \text { free }}\left(\mu^{\frac{1}{2}} r\right) .
$$

Therefore, the necklace $R_{\mu}^{(n)}(x, y)$ has the same asymptotic scaling. Hence, by (31), so does the eigenvector $\left(\begin{array}{l}v \\ u\end{array}\right)$, while the eigenvalue $\Omega$ scales asymptotically as $\mu$. 


\subsection{Rectangular necklaces}

When $R_{\mu}=R_{\mu}^{(1)}$ is a single pearl on the square $D=[-1,1]^{2}$, there are no eigenvalues of (31) with a positive real part, see Fig. 15(a). This is in agreement with the observed stability of $\psi_{\mathrm{sw}}^{(1)}=e^{i \mu z} R_{\mu}^{(1)}$ in Fig. 13(a).

Next, we investigate the stability of $\psi_{\mathrm{sw}}^{(2 \times 1)}=R_{\mu}^{(2 \times 1)}(x, y) e^{i \mu z}$, where $R_{\mu}^{(2 \times 1)}$ is a rectangular necklace with 2 pearls. Fig. 15(b) shows that there are no eigenvalues of $(31)$ with $\operatorname{Re}(\Omega)>0$ for $\mu_{\text {lin }} \leqslant \mu<\mu_{\text {cr }}$, and that there is a simple eigenvalue $\Omega_{1}(\mu)$ with $\operatorname{Re}\left(\Omega_{1}\right)>0$ for $\mu_{\text {cr }}<$ $\mu<\infty$, where $\mu_{\text {lin }}=-\frac{\pi^{2}}{2} \approx-4.9$, see (18), and $\mu_{\mathrm{cr}} \approx-4$. Hence, $\psi_{\mathrm{sw}}^{(2 \times 1)}$ is linearly stable for $\mu_{\text {lin }} \leqslant \mu<\mu_{\text {cr }}$ and unstable for $\mu_{\text {cr }}<\mu<\infty$. As noted, $R^{(2 \times 1)}$ satisfies the VK condition for all $\mu$, since it is constructed from 2 identical ground states, each of which satisfies the VK condition. This does not lead to a contradiction, however, since the VK condition applies to ground states, which is not the case for multi-pearl necklaces.

The change from stability to instability occurs as the necklace power exceeds the threshold power

$$
P_{\text {th }}^{\text {necklace }}(n=2 \times 1):=P\left(R_{\mu_{\text {cr }}}^{(2 \times 1)}\right) \approx 0.275 P_{\text {cr }},
$$

see Figure 16(b), i.e., when the power of each pearl is $\approx 0.14 P_{\text {cr }}$. For comparison, all singlepearl solitary waves with power below $P_{\text {cr }}$ are stable. From this perspective, 2-pearl necklace solitary waves are considerably less stable than single-pearl ones.
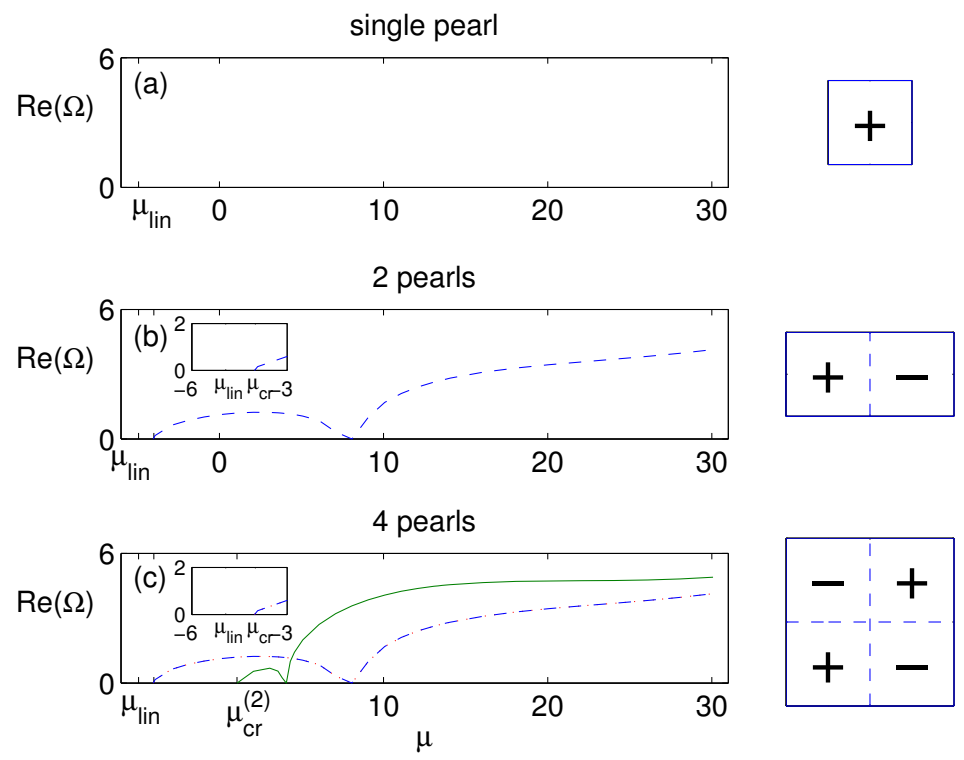

Figure 15: $\operatorname{Re}(\Omega)$ as a function of $\mu$ for all eigenvalues of (31) with a positive real part. Here $D$ is the rectangular domain shown on the right. (a) $R=R_{\mu}^{(1)}$. (b) $R=R_{\mu}^{(2 \times 1)}$. The dashed line corresponds to $\Omega_{1}$. (c) $R=R_{\mu}^{(2 \times 2)}$. The dashed blue and dotted red lines correspond to $\Omega_{1}$, and the solid green line to $\Omega_{2}$.

Following $(20 ; 14)$, in order to understand the instability dynamics of $\psi_{\mathrm{sw}}^{(2 \times 1)}$, in Fig 17 we plot the eigenfunction $f_{1}^{(2 \times 1)}:=\left(\begin{array}{c}v \\ u\end{array}\right)$ that corresponds to the unstable eigenvalue $\Omega_{1}$ of (31) with $R=R_{\mu}^{(2 \times 1)}$. We plot both the imaginary and real parts (and not just the 

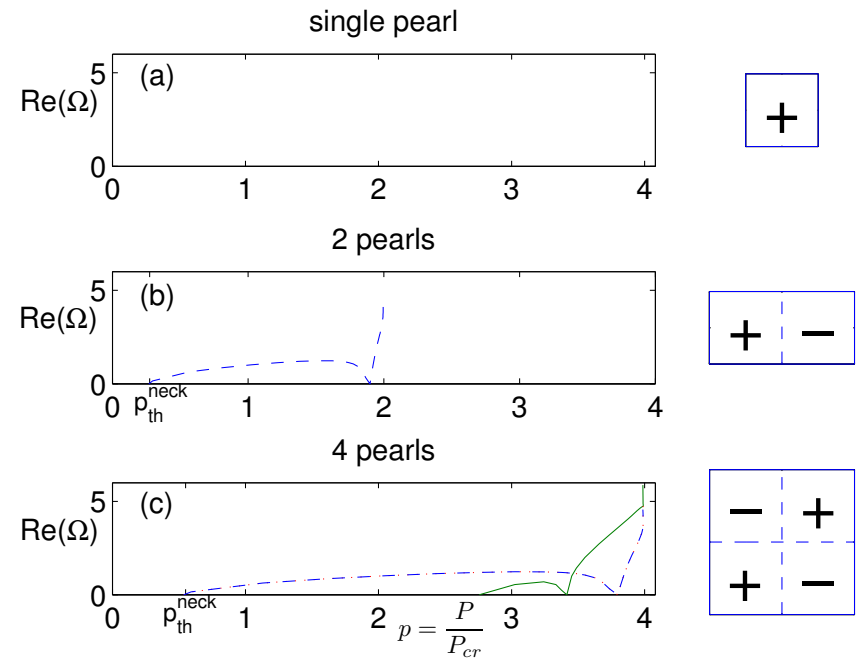

Figure 16: Same as Fig 15, where the $x$-coordinate is the fractional necklace power $p=P\left(R_{\mu}^{(n)}\right) / P_{\mathrm{cr}}$. Here $p_{\mathrm{th}}^{\text {neck }}:=P_{\mathrm{th}}^{\text {necklace }}(n) / P_{\mathrm{cr}}$ is $\approx 0.275$ in $(\mathrm{b})$ and $\approx 0.55$ in $(\mathrm{c})$.

absolute value) since we want to check whether $f_{1}^{(2 \times 1)}$ is symmetric or anti-symmetric. The unstable eigenfunction satisfies $f_{1}^{(2 \times 1)}(x, y)=f_{1}^{(2 \times 1)}(-x, y)$, in contrast to $R_{\mu}^{(2 \times 1)}$ that satisfies $R_{\mu}^{(2 \times 1)}(x, y)=-R_{\mu}^{(2 \times 1)}(-x, y)$. In other words, $f_{1}^{(2 \times 1)}$ is symmetric with respect to the interface $x=0$ between the pearls, whereas $R_{\mu}^{(2 \times 1)}$ is antisymmetric with respect to $x=0$. This shows that the instability is related to the breaking of the anti-symmetry between the two pearls. Since the addition of a symmetric perturbation to an anti-symmetric profile lowers one of the peaks while increasing the other, the instability evolves as power flows from one pearl to the other.
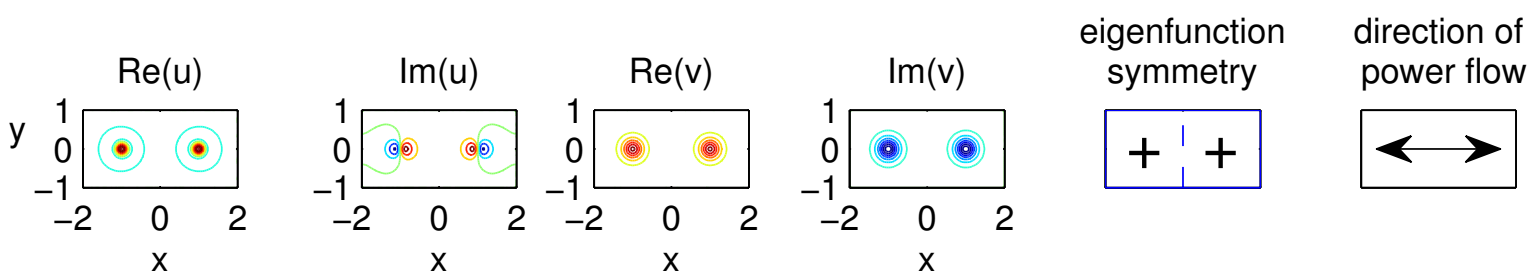

Figure 17: Real and imaginary parts of the eigenfunction $f_{1}^{(2 \times 1)}=\left(\begin{array}{l}v \\ u\end{array}\right)$ of (31) that corresponds to the unstable eigenvalue $\Omega_{1}(\mu=20)=3.4+4.4 i$. Here $D=[-2,2] \times[-1,1]$ and $R=R_{\mu=20}^{(2 \times 1)}$. The rightmost panel illustrates the directions in which the power flows as the instability evolves.

Next, we investigate the stability of $\psi_{\mathrm{sw}}^{(2 \times 2)}=R_{\mu}^{(2 \times 2)}(x, y) e^{i \mu z}$, where $R^{(2 \times 2)}$ is a square necklace with $2 \times 2$ pearls. Fig. $15(\mathrm{c})$ shows that $\psi_{\mathrm{sw}}^{(2 \times 2)}$ is stable for $\mu_{\text {lin }} \leqslant \mu<\mu_{\mathrm{cr}}$ and unstable for $\mu_{\mathrm{cr}}<\mu<\infty$, where $\mu_{\text {lin }}$ and $\mu_{\text {cr }}$ are as in the case of $R_{\mu}^{(2 \times 1)}$. Furthermore, $\psi_{\mathrm{sw}}^{(2 \times 2)}$ has an unstable eigenvalue $\Omega_{1}(\mu)$ of multiplicity 2 for $\mu_{\mathrm{cr}}<\mu<\infty$, whose value is 
the same as for $\psi_{\mathrm{sw}}^{(2 \times 1)}$, and an additional simple eigenvalue $\Omega_{2}(\mu)$ for $\mu_{\mathrm{cr}}^{(2)}<\mu<\infty$, where $\mu_{\mathrm{cr}}<\mu_{\mathrm{cr}}^{(2)} \approx 1$.
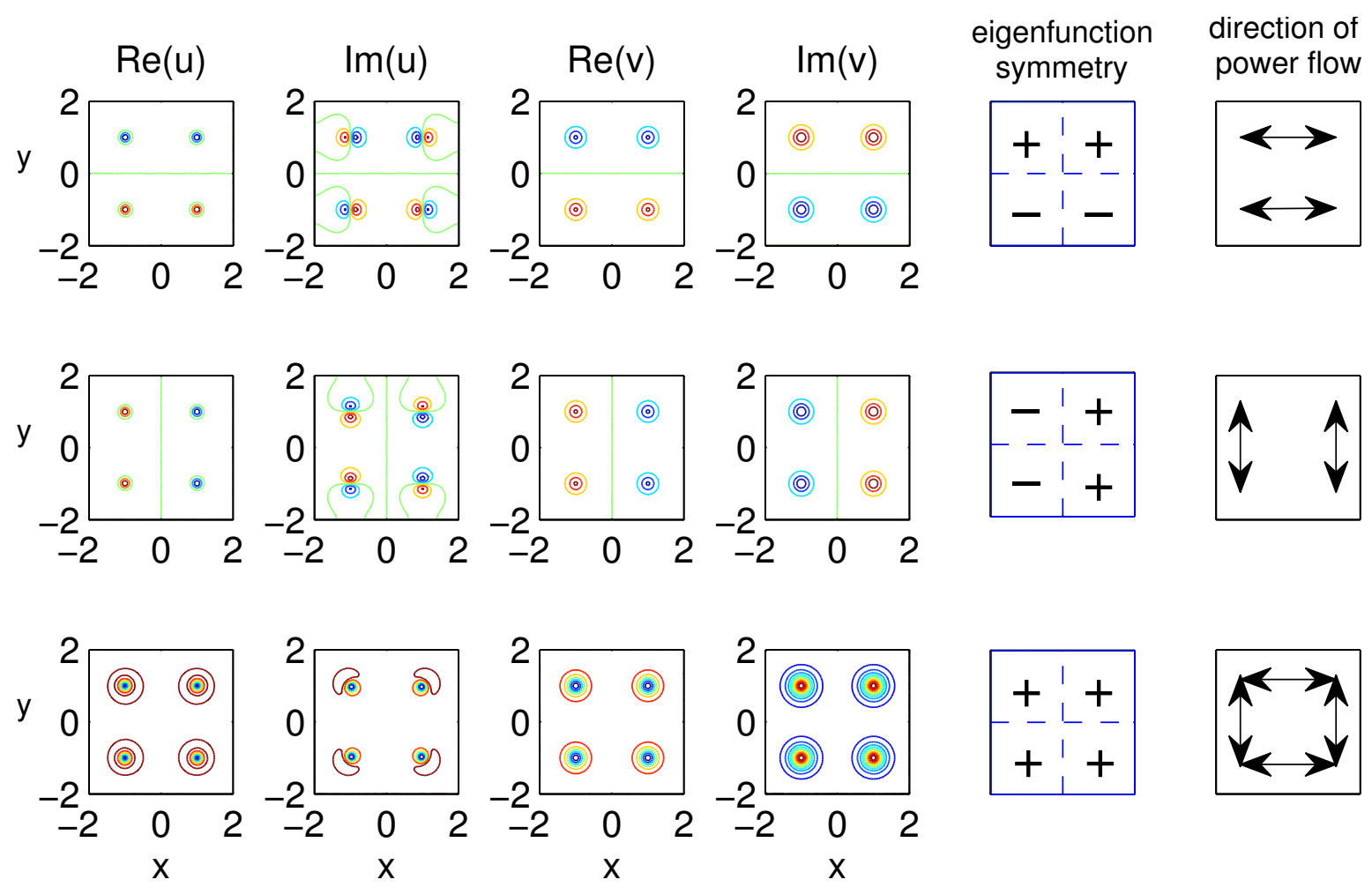

Figure 18: Same as Fig. 17 with $D=[-2,2]^{2}$ and $R=R_{\mu=20}^{(2 \times 2)}$. First row: The eigenfunction $f_{1,1}^{(2 \times 2)}$ of $\Omega_{1}$. Second row: The eigenfunction $f_{1,2}^{(2 \times 2)}$ of $\Omega_{1}$. Third row: The eigenfunction $f_{2}^{(2 \times 2)}$ of $\Omega_{2}$.

In Fig. 18 we plot the eigenfunctions that correspond to the unstable eigenvalues of $R_{\mu}^{(2 \times 2)}$ for $\mu=20$, which is in the regime $\mu_{\mathrm{cr}}^{(2)}<\mu<\infty$. One eigenfunction that corresponds to $\Omega_{1}(\mu=20)=3.4+4.4 i$ satisfies $f_{1,1}^{(2 \times 2)}(x, y)=f_{1,1}^{(2 \times 2)}(-x, y)$ and $f_{1,1}^{(2 \times 2)}(x, y)=-f_{1,1}^{(2 \times 2)}(x,-y)$. In contrast, $R_{\mu}^{(2 \times 2)}(x, y)=-R_{\mu}^{(2 \times 2)}(-x, y)$ and $R_{\mu}^{(2 \times 2)}(x, y)=-R_{\mu}^{(2 \times 2)}(x,-y)$. Thus, $f_{1,1}^{(2 \times 2)}$ breaks the anti-symmetry of $R_{\mu}^{(2 \times 2)}$ in the $x$-direction, but preserves it in the $y$-direction. It follows that there is a decoupling between the top and bottom pair of pearls as the instability evolves. In fact, as illustrated in Figure $19(\mathrm{a}), f_{1,1}^{(2 \times 2)}(x, y)$ is nothing but $f_{1}^{(2 \times 1)}(x, y)$ for $-2 \leq y \leq 0$, and $-f_{1}^{(2 \times 1)}(-x,-y)$ for $0 \leq y \leq 2$, where $f_{1}^{(2 \times 1)}$ is the unstable eigenfunction of $(31)$ with $R_{\mu}^{(2 \times 1)}$, which is plotted in Figure 17. This explain why the values of $\mu_{\mathrm{cr}}$ and $\Omega_{1}(\mu)$ for $R_{\mu}^{(2 \times 1)}$ and for $R_{\mu}^{(2 \times 2)}$ are identical, and also implies that

$$
P_{\mathrm{th}}^{\text {necklace }}(n=2 \times 2):=P\left(R_{\mu_{\mathrm{cr}}}^{(2 \times 2)}\right)=2 \cdot P_{\mathrm{th}}^{\text {necklace }}(n=2 \times 1) \approx 0.55 P_{\mathrm{cr}},
$$

see Figure $16(\mathrm{c})$, where $P_{\mathrm{th}}^{\text {necklace }}(n=2 \times 2)$ is the threshold power for instability of a $2 \times 2$ square necklace.

The second eigenfunction that corresponds to $\Omega_{1}(\mu=20)=3.4+4.4 i$ is a $90^{\circ}$ rotation of the first eigenfunction, i.e., $f_{1,2}^{(2 \times 2)}(x, y)$ is $f_{1}^{(2 \times 1)}(-y, x)$ for $-2 \leq x \leq 0$ and $-f_{1}^{(2 \times 1)}(y,-x)$ 
for $0 \leq x \leq 2$, see Figure 19(b). Thus, $f_{1,2}^{(2 \times 2)}$ preserves the antisymmetry in $x$, but breaks it in $y$. Hence, in the instability dynamics which is excited by this eigenfunction, there is a decoupling between the left and right pairs of pearls.

The unstable eigenfunction which corresponds to $\Omega_{2}(\mu=20)=4.7+4.6 i$, which is denoted by $f_{2}^{(2 \times 2)}$, satisfies $f_{2}^{(2 \times 2)}(x, y)=f_{2}^{(2 \times 2)}(-x, y)=f_{2}^{(2 \times 2)}(x,-y)=f_{2}^{(2 \times 2)}(-x,-y)$. Hence, it breaks the antisymmetry between all pearls. Therefore, the power flows between all 4 pearls.
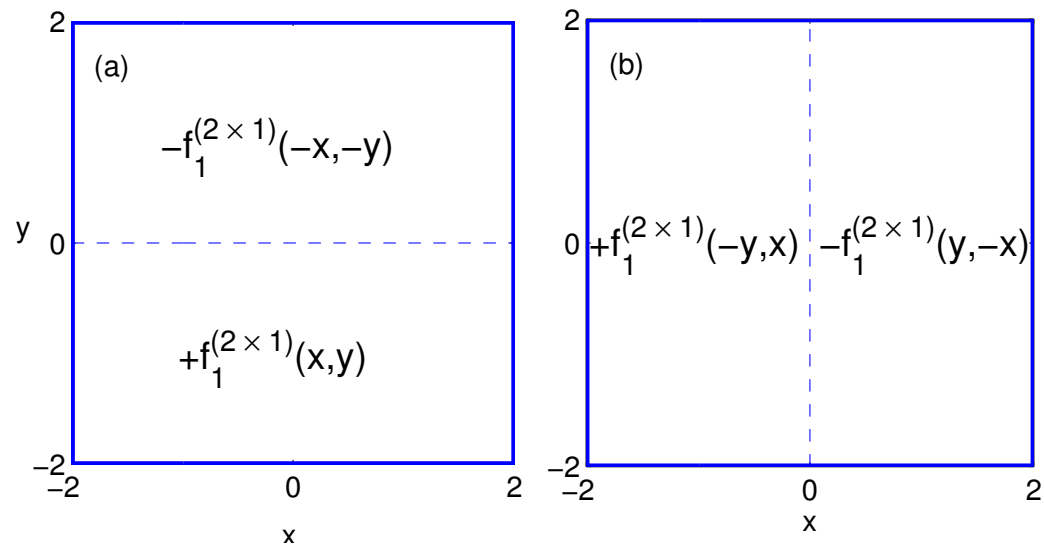

Figure 19: (a) Construction of $f_{1,1}^{(2 \times 2)}$ using $f_{1}^{(2 \times 1)}$. (b) Construction of $f_{1,2}^{(2 \times 2)}$ using $f_{1}^{(2 \times 1)}$. See details in text.

\subsubsection{Instability dynamics}

In free space, unstable solitary waves either collapse or scatter. On bounded domains, however, scattering is impossible. In addition, since $P_{\text {th }}^{\text {necklace }}(n=2 \times 2) \approx 0.55 P_{\text {cr }}$, see (33), then for $\mu$ slightly above $\mu_{\mathrm{cr}}$ we have that $P\left(R_{\mu}^{(2 \times 2)}\right)<P_{\mathrm{cr}}$, and so $\psi_{\mathrm{sw}}^{(2 \times 2)}$ cannot collapse under small perturbations. Indeed, the change from stability to instability of $\psi_{\mathrm{sw}}^{(2 \times 2)}$ is associated with the loss of the necklace structure, ${ }^{12}$ due to power flow between pearls, and not with collapse or scattering. To see this, in Fig. 20 we solve the NLS numerically with a perturbed $R_{\mu}^{(2 \times 2)}$ initial condition. In order to excite the unstable modes, we multiply the top-right pearl by 1.05 and leave the other three pearls unchanged. ${ }^{13}$ Fig. 20 shows that $\psi_{\mathrm{sw}}^{(2 \times 2)}$ preserves its necklace shape till $z=10$ for $\mu=\mu_{\mathrm{cr}}-\frac{1}{4}$, but disintegrates before $z=3$ for $\mu=\mu_{\mathrm{cr}}+\frac{1}{4}$. This confirms that there is a qualitative change in the necklace stability at $\mu_{\mathrm{cr}}$.

\footnotetext{
${ }^{12}$ i.e., the loss of antisymmetry between adjacent pearls.

${ }^{13}$ Unlike Figure 13, we do not test for stability by multiplying $\psi_{0}$ by 1.05 , since in that case $\psi_{0}$ remains antisymmetric with respect to the interfaces between the pearls, and hence so does $\psi$. Consequently, $\psi \equiv 0$ on the interfaces between pearls, which thus act as reflecting boundaries that prevent the pearls from interacting with each other. Since each pearl is stable, see Figure 13(a), the necklace remains stable under this antisymmetry-preserving perturbation (see Lemma 6).
} 

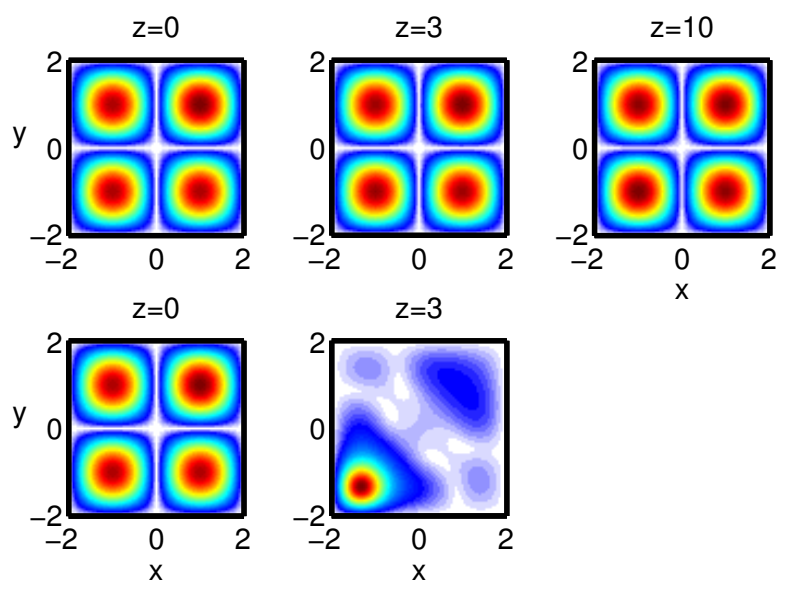

Figure 20: Solution of the NLS (3) on the domain $D=[-2,2]^{2}$ at different distances. Here $\psi_{0}=$ $R_{\mu}^{(2 \times 2)}(x, y) H(\theta)$, where $H(\theta)$ is given by (30). Top row: $\mu=\mu_{\mathrm{cr}}-\frac{1}{4}$. Bottom row: $\mu=\mu_{\mathrm{cr}}+\frac{1}{4}$. Here $\mu_{\mathrm{cr}} \approx-4$.

\subsection{Circular necklaces}

When $R_{\mu}=R_{\mu}^{(1)}$ is a single pearl on the quarter circle $\left\{0 \leq r \leq 1,0 \leq \theta \leq \frac{\pi}{2}\right\}$, there are no eigenvalues of (31) with a positive real part, see Fig. 21(a). This is in agreement with the observed stability of $\psi_{\mathrm{sw}}^{(1)}=e^{i \mu z} R_{\mu}^{(1)}$ in Fig. 13(b).

Next, we investigate the stability of $\psi_{\mathrm{sw}}^{(2)}=R_{\mu}^{(2)}(x, y) e^{i \mu z}$, where $R_{\mu}^{(2)}$ is a 2-pearl necklace on the semi-circle. Fig. 21(b) shows that there are no eigenvalues of (31) with $\operatorname{Re}(\Omega)>0$ for $\mu_{\text {lin }} \leqslant \mu<\mu_{\text {cr }}$, and there is a single eigenvalue $\Omega_{1}$ with $\operatorname{Re}\left(\Omega_{1}\right)>0$ for $\mu_{\text {cr }}<\mu<\infty$, where $\mu_{\mathrm{lin}}=-k_{2}^{2} \approx-26.4$, see $(21)$, and $\mu_{\mathrm{cr}} \approx-24.3$. Hence, $\psi_{\mathrm{sw}}^{(2)}$ is linearly stable for $\mu_{\mathrm{lin}} \leqslant \mu<\mu_{\mathrm{cr}}$ and unstable for $\mu_{\mathrm{cr}}<\mu<\infty .{ }^{14}$

The change from stability to instability occurs as the necklace power exceeds the threshold power

$$
P_{\text {th }}^{\text {necklace }}(n=2)=P\left(R_{\mu_{\mathrm{cr}}}^{(2)}\right) \approx 0.12 P_{\mathrm{cr}},
$$

see Figure 22(b), i.e., when the power of each pearl is $\approx 0.06 P_{\mathrm{cr}}$. Thus, the threshold power for instability of a 2-pearl necklace on the semi-circle is less than half of that on a rectangle, see (32). We have no explanation for this surprising observation.

In Figure 23 we plot the eigenfunction $f_{1}^{(2)}:=\left(\begin{array}{l}v \\ u\end{array}\right)$ that corresponds to the unstable eigenvalue $\Omega_{1}$ associated with $R_{\mu}^{(2)}$. The unstable eigenfunction satisfies $f_{1}^{(2)}(x, y)=$ $f_{1}^{(2)}(-x, y)$, in contrast to $R_{\mu}^{(2)}$, which satisfies $R_{\mu}^{(2)}(x, y)=-R_{\mu}^{(2)}(-x, y)$. This shows that the instability of $\psi_{\mathrm{sw}}^{(2)}$ is related to the breaking of the anti-symmetry between the two pearls.

\footnotetext{
${ }^{14}$ As noted, $R^{(2)}$ satisfies the VK condition for all $\mu$, since it is constructed from two identical ground states, each of which satisfies the VK condition. This does not lead to a contradiction, however, since the VK condition applies to positive solutions, which is not the case for multi-pearl necklaces.
} 

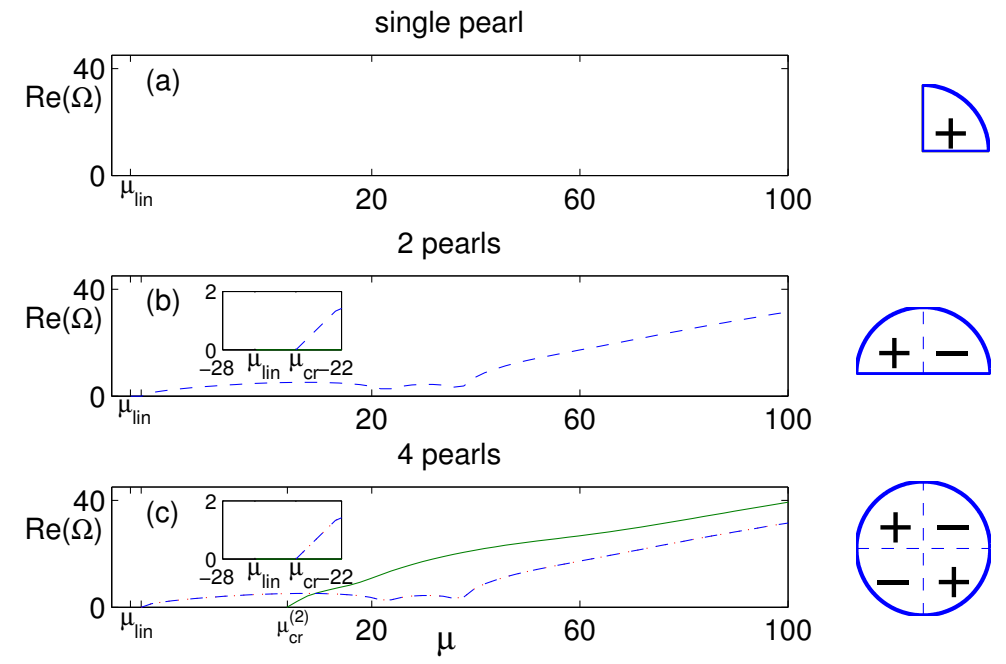

Figure 21: Same as Figure 15, where $D$ is the sector of a circle shown on the right. (a) $R=R_{\mu}^{(1)}$, (b) $R=R_{\mu}^{(2)}$, (c) $R=R_{\mu}^{(4)}$.
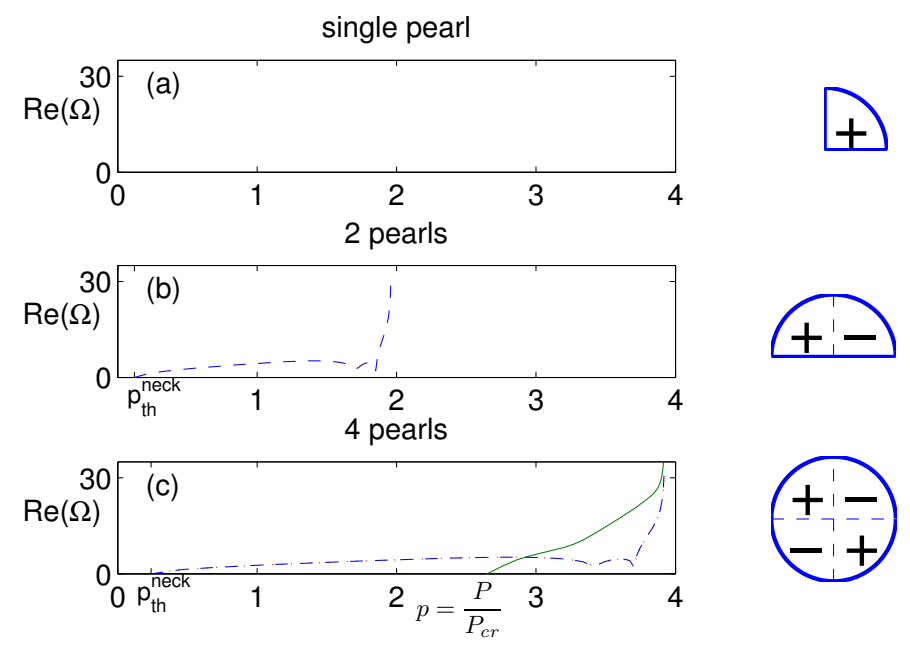

Figure 22: Same as Fig 21, where the $x$-coordinate is the fractional necklace power $p=P\left(R_{\mu}^{(n)}\right) / P_{\mathrm{cr}}$. Here $p_{\mathrm{th}}^{\text {neck }}:=P_{\mathrm{th}}^{\text {necklace }}(n) / P_{\mathrm{cr}}$ is $\approx 0.12$ in $(\mathrm{b})$ and $\approx 0.24$ in $(\mathrm{c})$.

Thus, the instability evolves as power flows from one pearl to the other.

Next, we investigate the stability of $\psi_{\mathrm{sw}}^{(4)}=R_{\mu}^{(4)}(x, y) e^{i \mu z}$, where $R^{(4)}$ is a circular necklace with 4 pearls. Fig. 21(c) shows that $\psi_{\mathrm{sw}}^{(4)}$ is linearly stable for $\mu_{\text {lin }} \leqslant \mu<\mu_{\mathrm{cr}}$ and unstable for $\mu_{\mathrm{cr}}<\mu<\infty$, where $\mu_{\mathrm{lin}}$ and $\mu_{\mathrm{cr}}$ are as in the case of $R_{\mu}^{(2)}$. Furthermore, $\psi_{\mathrm{sw}}^{(4)}$ has an unstable eigenvalue $\Omega_{1}$ of multiplicity 2 for $\mu_{\mathrm{cr}}<\mu<\infty$, whose value is the same as for $\psi_{\mathrm{sw}}^{(2)}$, and an additional simple eigenvalue $\Omega_{2}$ for $\mu_{\mathrm{cr}}^{(2)}<\mu<\infty$, where $\mu_{\mathrm{cr}}<\mu_{\mathrm{cr}}^{(2)} \approx 4$.

In Fig. 24 we plot the eigenfunctions that correspond to the unstable eigenvalues for $\mu=12$, which is in the regime $\mu_{\mathrm{cr}}^{(2)}<\mu<\infty$. As in the rectangular case, one eigenfunction that corresponds to $\Omega_{1}(\mu=12)=5+14 i$ satisfies $f_{1,1}^{(4)}(x, y)=f_{1,1}^{(4)}(-x, y)$ and $f_{1,1}^{(4)}(x, y)=$ 


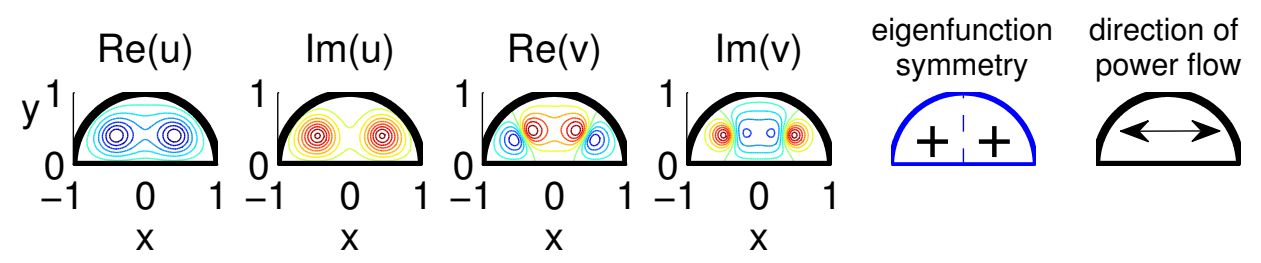

Figure 23: Same as Fig. 17 with $D$ being the upper half circle, $R=R_{\mu=12}^{(2)}$, and $\Omega_{1}(\mu=12)=5+14 i$.

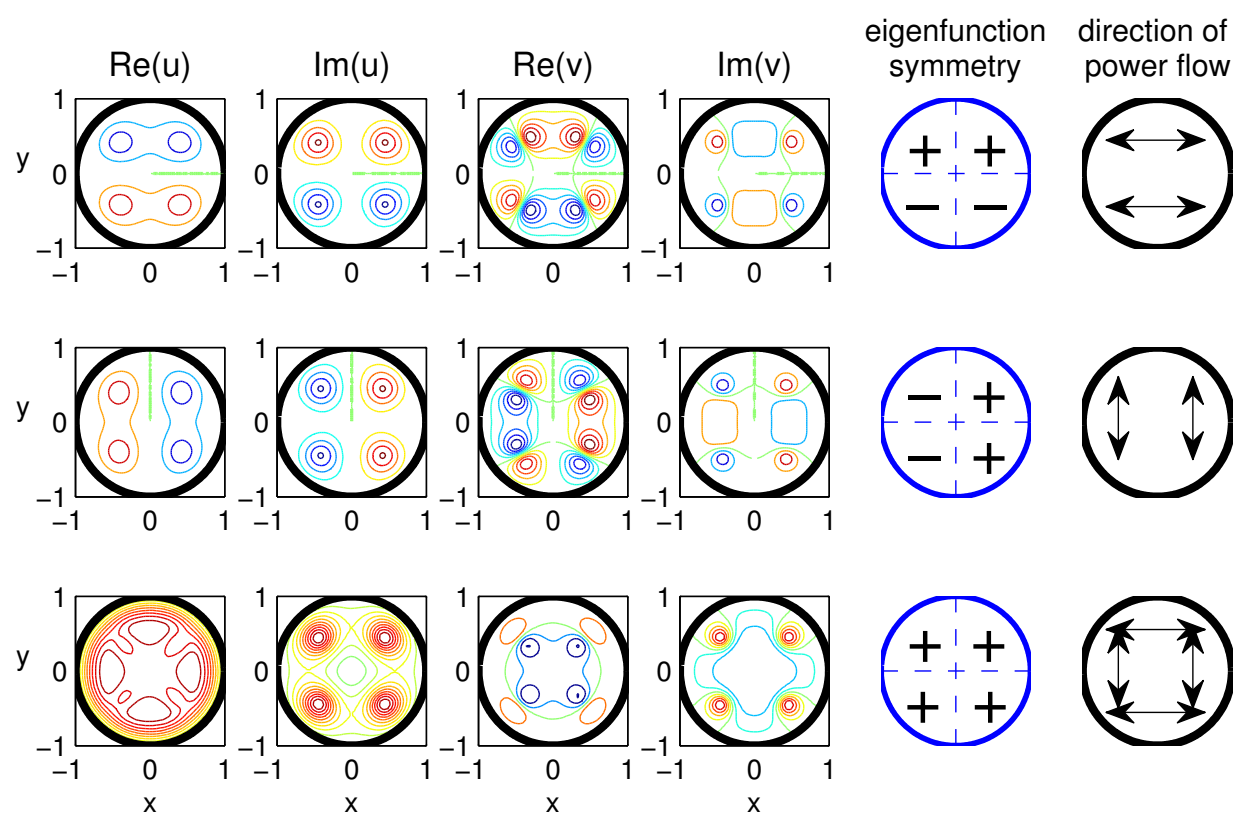

Figure 24: Same as Fig. 17, with $D$ being the unit circle and $R=R_{\mu=12}^{(4)}$. First row: The eigenfunction $f_{1,1}^{(4)}$ of $\Omega_{1}$. Second row: The eigenfunction $f_{1,2}^{(4)}$ of $\Omega_{1}$. Third row: The eigenfunction $f_{2}^{(4)}$ of $\Omega_{2}$.

$-f_{1,1}^{(4)}(x,-y)$. In contrast, $R_{\mu}^{(4)}(x, y)=-R_{\mu}^{(4)}(-x, y)$ and $R_{\mu}^{(4)}(x, y)=-R_{\mu}^{(4)}(x,-y)$. Thus, $f_{1,1}^{(4)}$ breaks the anti-symmetry of $R_{\mu}^{(4)}$ in the $x$-direction, but preserves it in the $y$-direction. Hence, there is decoupling between the top and bottom pair of pearls as the instability evolves. In fact, as illustrated in Figure $25(\mathrm{a}), f_{1,1}^{(4)}(x, y)$ is nothing but $f_{1}^{(2)}(x, y)$ for $0 \leq y \leq$ 1 , and $-f_{1}^{(2)}(-x, y)$ for $-1 \leq y \leq 0$, where $f_{1}^{(2)}$ is the unstable eigenfunction of (31) with $R^{(2)}$, which is plotted in Figure 23. This explain why the values of $\mu_{\mathrm{cr}}$ and $\Omega_{1}(\mu)$ for $R^{(2)}$ and for $R_{\mu}^{(4)}$ are identical, and also implies that the threshold power for instability of a 4-pearl circular necklace satisfies

$$
P_{\mathrm{th}}^{\text {necklace }}(n=4)=2 P_{\mathrm{th}}^{\text {necklace }}(n=2) \approx 0.24 P_{\mathrm{cr}},
$$

see Figure 22(c).

The second eigenfunction that corresponds to $\Omega_{1}(\mu=12)=5+14 i$ is a $90^{\circ}$ rotation of the first eigenfunction, i.e., $f_{1,2}^{(4)}(x, y)$ is $-f_{1}^{(2)}(y,-x)$ for $-1 \leq x \leq 0$, and $f_{1}^{(2)}(-y, x)$ 
for $0 \leq x \leq 1$, see Figure 25(b). For this eigenfunction, there is decoupling between the instability dynamics of the left and right pairs of pearls. The unstable eigenfunction which corresponds to $\Omega_{2}(\mu=12)=6.7+19 i$ is denoted by $f_{2}^{(4)}$. It satisfies $f_{2}^{(4)}(x, y)=f_{2}^{(4)}(-x, y)=$ $f_{2}^{(4)}(x,-y)=f_{2}^{(4)}(-x,-y)$, i.e., it breaks the antisymmetry between all pearls. Therefore, the power flows between all 4 pearls.
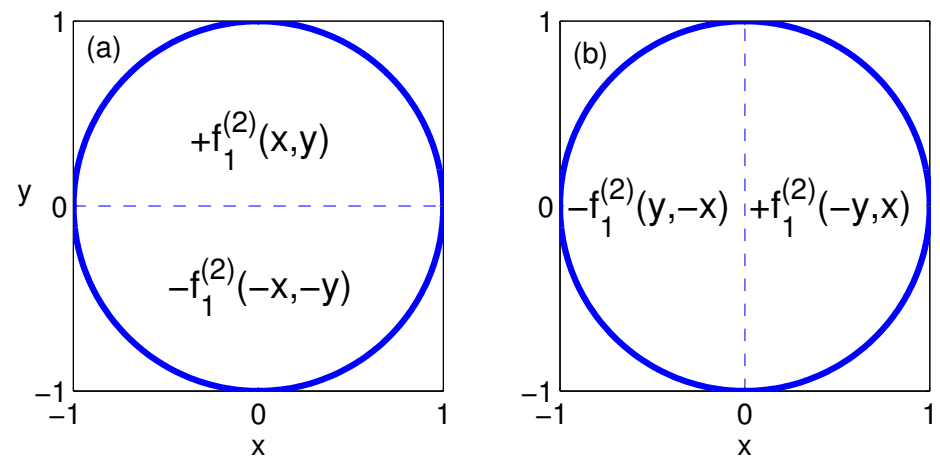

Figure 25: (a) Construction of $f_{1,1}^{(4)}$ using $f_{1}^{(2)}$. (b) Construction of $f_{1,2}^{(4)}$ using $f_{1}^{(2)}$. See details in the text.

\subsubsection{Instability dynamics}

As in the rectangular case, the change from stability to instability of $\psi_{\mathrm{sw}}^{(4)}$ is associated with the loss of the necklace structure due to power flow between pearls, and not with collapse or scattering. To see this, we solve the NLS numerically with a perturbed $R_{\mu}^{(4)}$ initial condition. In order to excite the unstable modes, we multiply the top-left pearl by 1.05 and leave the other three pearls unchanged. Fig. 26 shows that $\psi_{\mathrm{sw}}^{(4)}$ preserves its necklace shape till $z=10$ for $\mu=\mu_{\mathrm{cr}}-1$, but that it disintegrates before $z=3$ for $\mu=\mu_{\mathrm{cr}}-1$. This confirms that there is a qualitative change in the necklace stability at $\mu_{\mathrm{cr}}$.

\subsection{Annular necklaces}

When $R_{\mu}=R_{\mu}^{(1)}$ is a single pearl on the quarter-annulus $\left\{0.5 \leq r \leq 1,0 \leq \theta \leq \frac{\pi}{2}\right\}$, there are no eigenvalues of (31) with a positive real part, see Fig. 27(a). This is in agreement with the observed stability of $\psi_{\mathrm{sw}}^{(1)}=e^{i \mu z} R_{\mu}^{(1)}$ in Fig. 13(c).

Next, we investigate the stability of $\psi_{\mathrm{sw}}^{(2)}=e^{i \mu z} R_{\mu}^{(2)}(x, y)$, where $R_{\mu}^{(2)}$ is a 2-pearl necklace on the half-annulus $\{0.5 \leq r \leq 1,0 \leq \theta \leq \pi\}$. Fig. 27(b) shows that there are no eigenvalues of $(31)$ with $\operatorname{Re}(\Omega)>0$ for $\mu_{\text {lin }} \leqslant \mu<\mu_{\text {cr }}$ and for $\mu_{2} \leqslant \mu<\mu_{4}$, and there is a single eigenvalue $\Omega_{1}$ with $\operatorname{Re}\left(\Omega_{1}\right)>0$ for $\mu_{\mathrm{cr}}<\mu<\mu_{2}$ and for $\mu_{4}<\mu<\infty$, where $\mu_{\text {lin }}=-k_{2}^{2} \simeq-46.4$, see $(23), \mu_{\mathrm{cr}} \approx-43.6, \mu_{2} \approx 23$, and $\mu_{4} \approx 117$. Hence, $\psi_{\mathrm{sw}}^{(2)}$ is linearly stable for $\mu_{\text {lin }} \leqslant \mu<\mu_{\text {cr }}$ and $\mu_{2}<\mu<\mu_{4}$, and unstable for $\mu_{\text {cr }}<\mu<\mu_{2}$ and $\mu_{4}<\mu<\infty$.

The initial change from stability to instability occurs as the necklace power exceeds

$$
P_{\mathrm{th}}^{\text {necklace }}\left(R_{\mu}^{(2)}\right)=P\left(R_{\mu_{\mathrm{cr}}}^{(2)}\right) \approx 0.12 P_{\mathrm{cr}}
$$



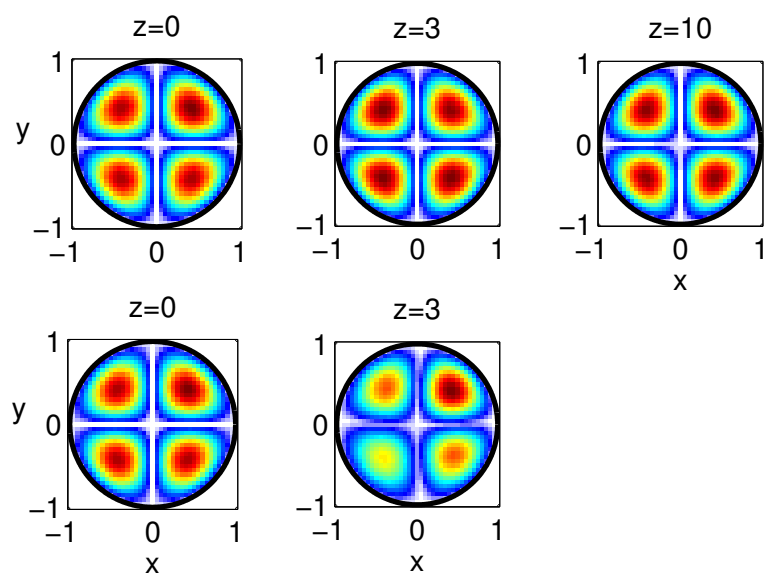

Figure 26: Same as Figure 20 on a circular domain. Top row: $\mu=\mu_{\mathrm{cr}}-1$. Bottom row: $\mu=\mu_{\mathrm{cr}}+1$. Here $\mu_{\mathrm{cr}} \approx-24.3$.

see Figure $28(\mathrm{~b})$, i.e., when the power of each pearl is $\approx 0.06 P_{\mathrm{cr}}$. Thus, the threshold power for instability of a two-pearl necklace on the half-annulus is the same as that on the halfcircle, see (34). This is surprising, since the necklace instability is associated with power transfer between the pearls, and so the hole could be expected to have a stabilizing effect.

The behavior of $\operatorname{Re}\left(\Omega_{1}\right)$ for $\mu_{\text {lin }}<\mu<\mu_{4}$ is different from that for a two-pearl necklace on a rectangle and on a semi-circle, see Figures 15(b) and 21(b), respectively. It is, however, similar to that for a two-pearl one-dimensional necklace, see Figure 33(b). Intuitively, this is because the Dirichlet boundary conditions at $r=r_{\min }$ and $r=r_{\max }$ "clamp" the necklace profile in the radial direction. Consequently, the dependence of $R_{\mu}^{(1)}$ on $\mu$ is predominantly one-dimensional. Once $\mu>\mu_{4}$, however, the pearls become so narrow that they "do not feel" the radial walls. Therefore, they approach the free-space two-dimensional ground state $R_{\mu, 2 \mathrm{D}}^{(1), \text { free }}$. Hence, $\operatorname{Re}\left(\Omega_{1}\right)$ increases linearly in $\mu$ (see Section 5.2).

We now discuss the stability of $\psi_{\mathrm{sw}}^{(4)}=R_{\mu}^{(4)}(r, \theta) e^{i \mu z}$, where $R_{\mu}^{(4)}$ is an annular necklace with 4 pearls, see Sec. 4.3.2. Fig. $27(\mathrm{c})$ shows that $\psi_{\mathrm{sw}}^{(4)}$ is linearly stable for $\mu_{\mathrm{lin}} \leqslant \mu<\mu_{\mathrm{cr}}$ and $\mu_{2} \leqslant \mu<\mu_{3}$, and unstable for $\mu_{\mathrm{cr}}<\mu<\mu_{2}$ and $\mu_{3}<\mu<\infty$, where $\mu_{\text {lin }}, \mu_{\mathrm{cr}}$, and $\mu_{2}$ are the same as for $\psi_{\mathrm{sw}}^{(2)}$, and $\mu_{3} \approx 73 .^{15}$

In Fig. 29 we plot the eigenfunctions that correspond to the unstable eigenvalues for $\mu=-28$. One eigenfunction that corresponds to $\Omega_{1}(\mu=-28)=3+7.6 i$ satisfies $f_{1,1}^{(4)}(x, y)=$ $f_{1,1}^{(4)}(-x, y)$ and $f_{1,1}^{(4)}(x, y)=-f_{1,1}^{(4)}(x,-y)$. In contrast, $R_{\mu}^{(4)}(x, y)=-R_{\mu}^{(4)}(-x, y)$ and $R_{\mu}^{(4)}(x, y)=$ $-R_{\mu}^{(4)}(x,-y)$. Thus, $f_{1,1}^{(4)}$ breaks the anti-symmetry of $R_{\mu}^{(4)}$ in the $x$-direction, but preserves it in the $y$-direction. Hence, there is decoupling between the top and bottom pair of pearls as the instability evolves. As in the rectangular and circular cases, $f_{1,1}^{(4)}(x, y)$ is nothing but $f_{1}^{(2)}(x, y)$ for $-1 \leq y \leq 0$, and $-f_{1}^{(2)}(-x,-y)$ for $0 \leq y \leq 1$ see Fig-

\footnotetext{
${ }^{15}$ The upper limit of the second stability regime is smaller than in the two-pearl case (i.e., $\mu_{3}<\mu_{4}$ ), since it is determined by the unstable eigenfunction $f_{2}^{(4)}$ which corresponds to $\Omega_{2}$.
} 


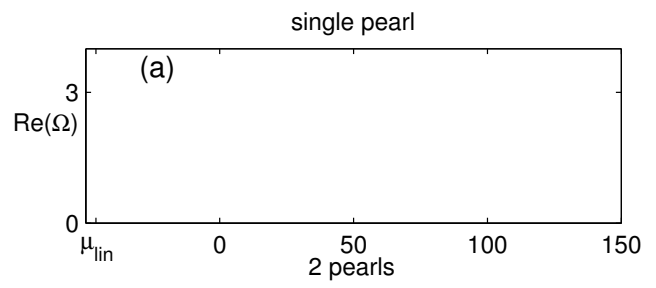

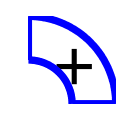
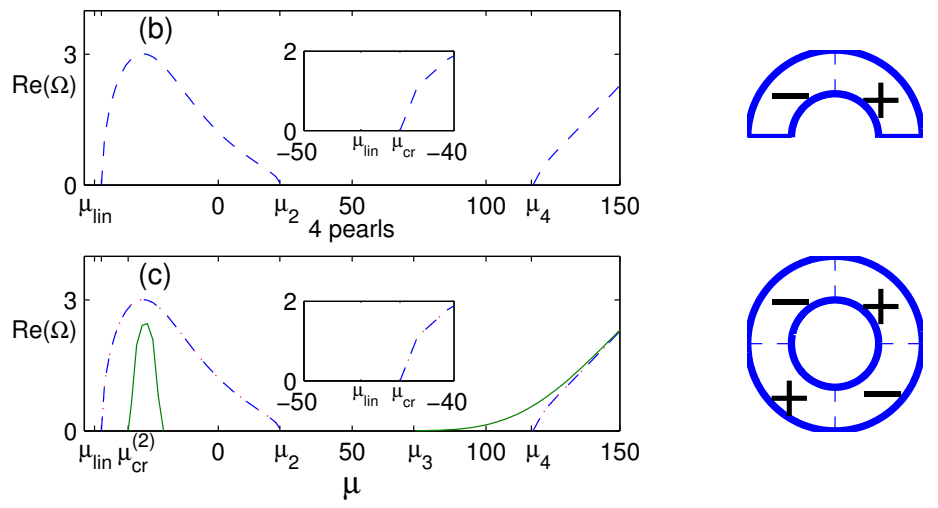

Figure 27: Same as Figure 15, where $R=R_{\mu}^{(4)}$, and $D$ is the sector of the annulus $0.5<r<1$ shown on the right.
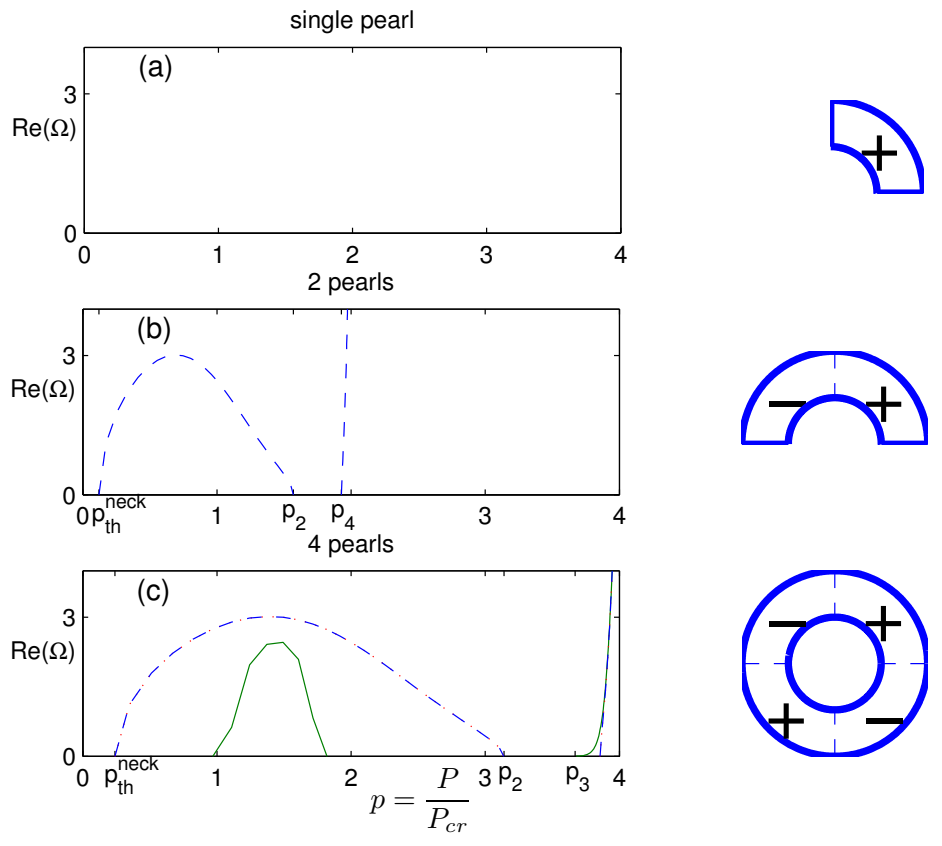

Figure 28: Same as Fig 27, where the $x$-coordinate is the fractional necklace power $p=P\left(R_{\mu}^{(n)}\right) / P_{\mathrm{cr}}$. Here $p_{\mathrm{th}}^{\text {neck }}=P_{\mathrm{th}}^{\text {necklace }}(n) / P_{\mathrm{cr}}$ and $p_{i}:=P\left(R_{\mu_{i}}^{(n)}\right) / P_{\mathrm{cr}}$.

ure 30(a), where $f_{1}^{(2)}$ is the unstable eigenfunction of (31) for a two-pearl necklace on the half-annulus. The second eigenfunction that corresponds to $\Omega_{1}(\mu=-28)=3+7.6 i$ is a $90^{\circ}$ rotation of the first eigenfunction, i.e., $f_{1,2}^{(4)}(x, y)$ is $f_{1}^{(2)}(-y, x)$ for $-1 \leq x \leq 0$, and 
$-f_{1}^{(2)}(y,-x)$ for $0 \leq x \leq 1$, see Figure $30(\mathrm{~b})$. For this eigenfunction, there is decoupling between the instability dynamics of the left and right pair of pearls. The unstable eigenfunction that corresponds to $\Omega_{2}(\mu=-28)=2.5+16 i$ is denoted by $f_{2}^{(4)}$. It satisfies $f_{2}^{(4)}(x, y)=f_{2}^{(4)}(-x, y)=f_{2}^{(4)}(x,-y)=f_{2}^{(4)}(-x,-y)$, i.e., it breaks the antisymmetry between all pearls. Therefore, the power flows between all 4 pearls.

The initial threshold power for the 4-pearl annular necklace instability is

$$
P_{\text {th }}^{\text {necklace }}(n=4)=P\left(R_{\mu_{\mathrm{cr}}}^{(4)}\right) \approx 0.24 P_{\mathrm{cr}},
$$

see Figure 28(c), which is the same as in the circular case, see (35). As noted, this result is surprising, as one could expect the hole to stabilize the necklace.
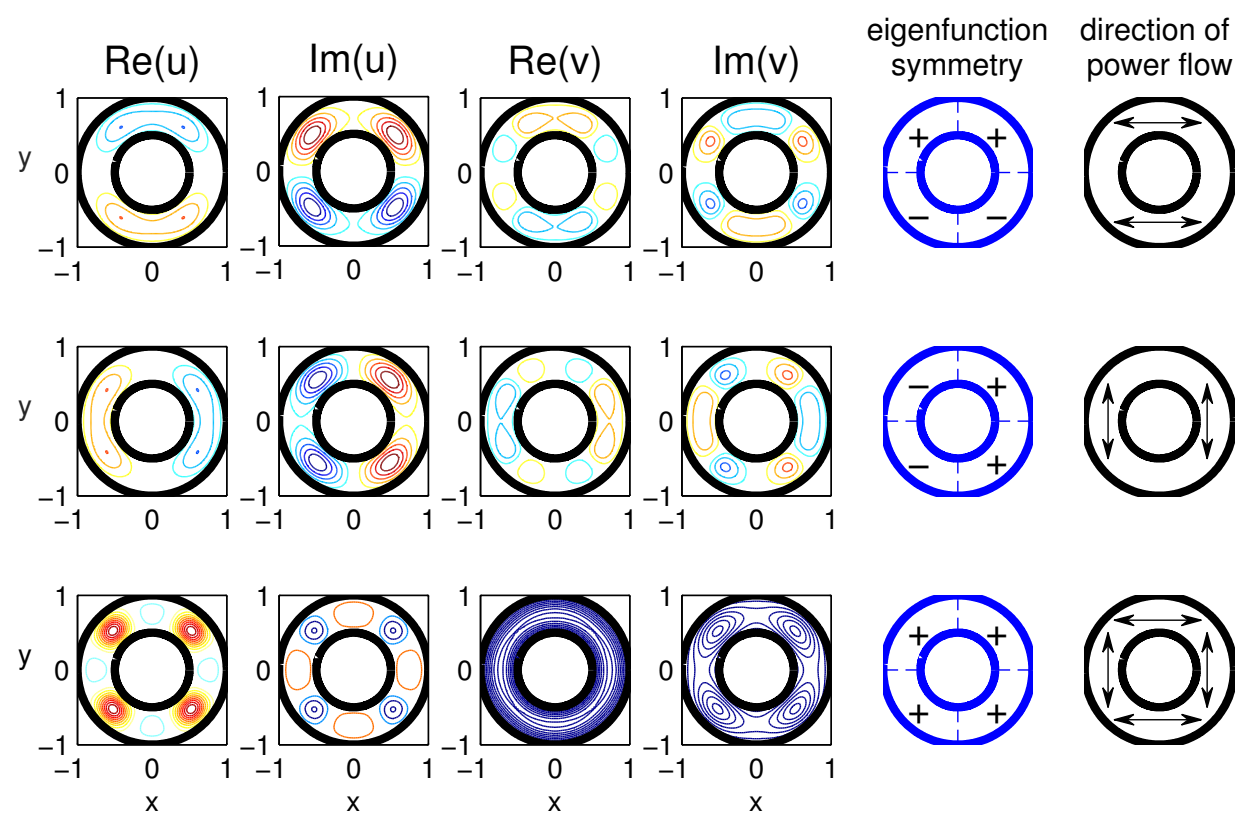

Figure 29: Same as Fig. 24 for the annular domain $D=\{0.5 \leq r \leq 1\}$ and $R=R_{\mu=-28}^{(4)}$.

As in the rectangular and circular cases, the initial change from stability to instability of $\psi_{\mathrm{sw}}^{(4)}$ is associated with the loss of the necklace structure due to power flow between pearls, and not with collapse or scattering. To see this, we solve the NLS numerically with a perturbed $R_{\mu}^{(4)}$ initial condition. In order to excite the unstable modes, we multiply the upper-right pearl by 1.05 and leave the other three pearls unchanged. Fig. 31 shows that $\psi_{\mathrm{sw}}^{(4)}$ preserves its necklace shape till $z=10$ for $\mu=\mu_{\mathrm{cr}}-1$, but disintegrates before $z=3$ for $\mu=\mu_{\mathrm{cr}}+1$. This confirms that there is a qualitative change in the necklace stability at $\mu_{\mathrm{cr}}$.

\subsubsection{Second stability regime}

Unlike the rectangular and circular cases, there is a second regime in which the annular necklace becomes linearly stable. This regime is $\mu_{2}<\mu<\mu_{3}$, see Figure 27(c), which corresponds to

$$
p_{2} P_{\mathrm{cr}}<P\left(R_{\mu}^{(4)}\right)<p_{3} P_{\mathrm{cr}}, \quad p_{2}:=\frac{P\left(R_{\mu_{2}}^{(4)}\right)}{P_{\mathrm{cr}}} \approx 3.1, \quad p_{3}:=\frac{P\left(R_{\mu_{3}}^{(4)}\right)}{P_{\mathrm{cr}}} \approx 3.7,
$$



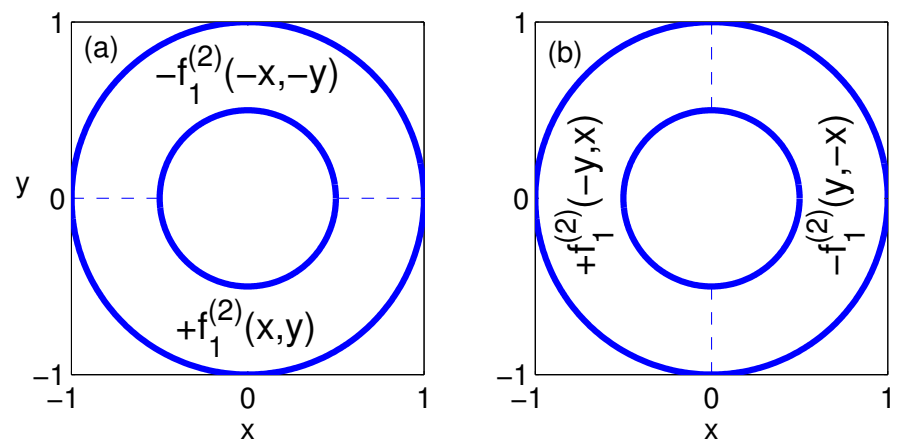

Figure 30: (a) Construction of $f_{1,1}^{(4)}$ using $f_{1}^{(2)}$. (b) Construction of $f_{1,2}^{(4)}$ using $f_{1}^{(2)}$. See details in text.

see Figure 28(c). In this regime the hole does stabilize the necklace. Indeed, Fig. 32 shows that when perturbed by $5 \%$ random noise, $\psi_{\mathrm{sw}}^{(4)}$ becomes unstable and collapses around $z=11.3$ for $\mu=10$, but remains stable until $z=15$ for $\mu=50$, where $P\left(R_{\mu=10}^{(4)}\right) \approx 2.9 P_{\mathrm{cr}}$ and $P\left(R_{\mu=50}^{(4)}\right) \approx 3.5 P_{\mathrm{cr}}$.
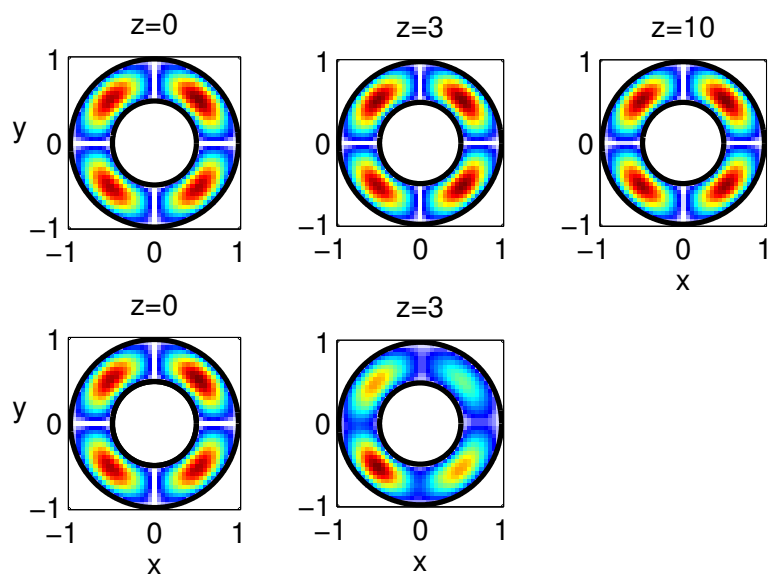

Figure 31: Same as Figure 20 for the annular domain $0.5 \leq r \leq 1$. Top row: $\mu=\mu_{\mathrm{cr}}-1$. Bottom row: $\mu=\mu_{\mathrm{cr}}+1$. Here $\mu_{\mathrm{cr}} \approx-43.6$.

\subsection{One-dimensional necklaces}

We now consider the stability of necklace solutions of the one-dimensional NLS (26). We simultaneously consider the subcritical case $\sigma=1$ and the critical case $\sigma=2$, in order to emphasize that, as far as necklace stability is concerned, the subcritical and critical cases are more similar than different. 

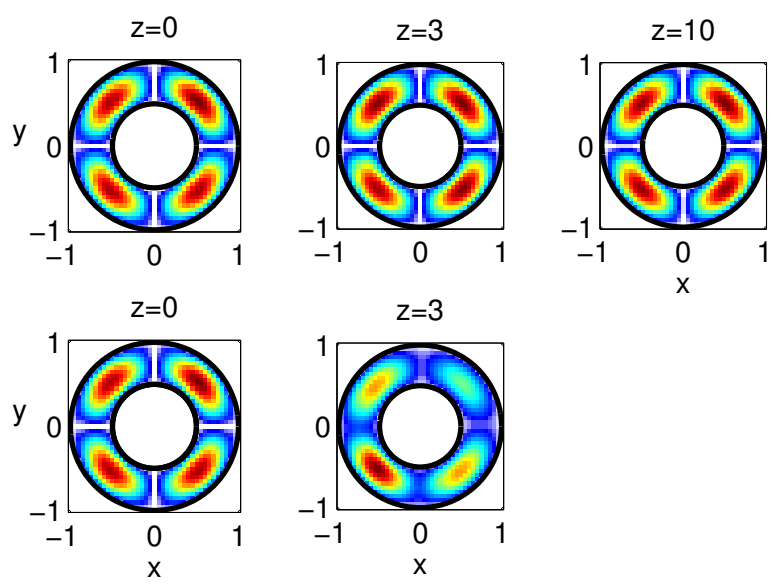

Figure 32: Same as Fig. 31 for $\psi_{0}=(1+0.05 \cdot \operatorname{noise}(x, y)) R_{\mu}^{(4)}$, where noise $(x, y)$ is uniformly distributed in $[-1,1]$. Top row: $\mu=10$. Bottom row: $\mu=50$.

Fukuizumi et al. (10) rigorously proved that when $0<\sigma \leq 2$ and $R_{\mu}^{(1)}$ is a single pearl on the interval $[-1,1]$, the solitary waves $\psi_{\mathrm{sw}}^{(1)}(z, x)=e^{i \mu z} R_{\mu}^{(1)}(x)$ of $(26)$ are stable for all $\mu_{\text {lin }}<\mu<\infty$. Indeed, there are no eigenvalues of (31) with a positive real part, see Fig. 33(a) and $34(\mathrm{a}){ }^{16}$

When $R=R_{\mu}^{(2)}$, a one-dimensional necklace with 2 pearls, there are no eigenvalues of (31) with $\operatorname{Re}(\Omega)>0$ for $\mu_{\text {lin }} \leqslant \mu<\mu_{\mathrm{cr}}$, and there is a single eigenvalue $\Omega_{1}$ with $\operatorname{Re}\left(\Omega_{1}\right)>0$ for $\mu_{\mathrm{cr}}<\mu<\infty$, see Fig. 33(b) and 34(b), where $\mu_{\text {lin }}=-\frac{\pi^{2}}{4} \approx-2.5$, see $(29), \mu_{\mathrm{cr}} \approx-1.56$ for $\sigma=1$, and $\mu_{\mathrm{cr}} \approx-2.16$ for $\sigma=2$. Hence, $\psi_{\mathrm{sw}}^{(2)}=R_{\mu}^{(2)}(x) e^{i \mu z}$ is linearly stable for $\mu_{\text {lin }} \leqslant \mu<\mu_{\text {cr }}$ and unstable for $\mu_{\text {cr }}<\mu<\infty$.

The eigenfunction $f_{1}^{(2)}:=\left(\begin{array}{c}v \\ u\end{array}\right)$ that corresponds to the unstable eigenvalue $\Omega_{1}$ associated with $R_{\mu}^{(2)}$ satisfies $f_{1}^{(2)}(x)=f_{1}^{(2)}(-x)$, see Figures 35 and 36, in contrast to $R_{\mu}^{(2)}$, which satisfies $R_{\mu}^{(2)}(x)=-R_{\mu}^{(2)}(-x)$. Thus, the instability of $\psi_{\mathrm{sw}}^{(2)}$ is related to the breaking of the anti-symmetry between the two pearls, and it evolves as power flows from one pearl to the other. As in the two-dimensional case, the necklace instability in unrelated to collapse. This is obvious in the case of the one-dimensional cubic NLS, which is subcritical and thus does not admit blowup solutions. In the one-dimensional quintic NLS, which is critical, the change from stability to instability occurs as the power of $R_{\mu}^{(2)}$ exceeds $P_{\mathrm{th}}^{\text {necklace }}\left(R_{\mu}^{(2)}\right)=P\left(R_{\mu_{\mathrm{cr}}}^{(2)}\right) \approx 0.25 P_{\mathrm{cr}},{ }^{17},{ }^{18}$ which is well below the critical power for collapse.

\footnotetext{
${ }^{16}$ In the one-dimensional case $L_{+}:=\frac{\partial^{2}}{\partial x^{2}}-\mu+(2 \sigma+1)|R|^{2}$ and $L_{-}:=\frac{\partial^{2}}{\partial x^{2}}-\mu+|R|^{2}$.

${ }^{17}$ i.e., when the power of each pearl is $\approx \frac{1}{8} P_{\text {cr. }}$.

${ }^{18}$ This result was already observed in (10).
} 

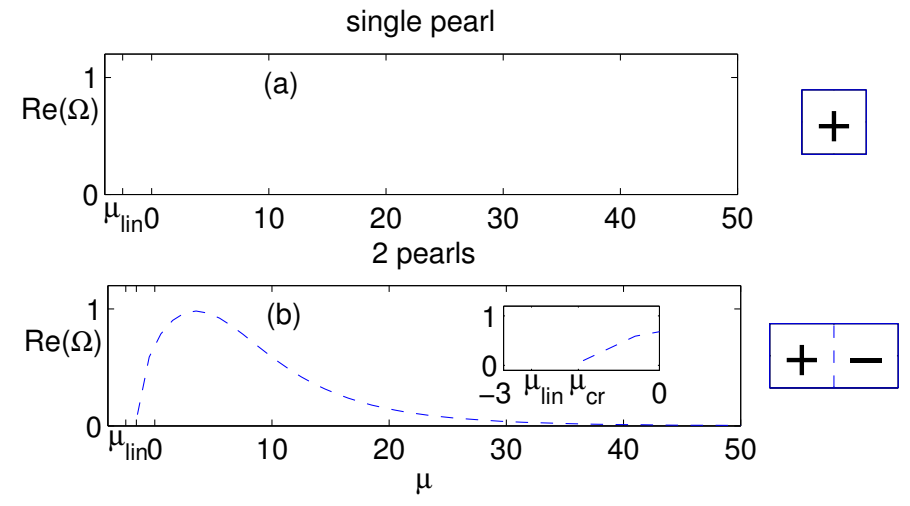

Figure 33: Same as Figure 15 for the one-dimensional NLS with $\sigma=1$. (a) $D=[-1,1]$ and $R=R_{\mu}^{(1)}$. (b) $D=[-2,2]$ and $R=R_{\mu}^{(2)}$.
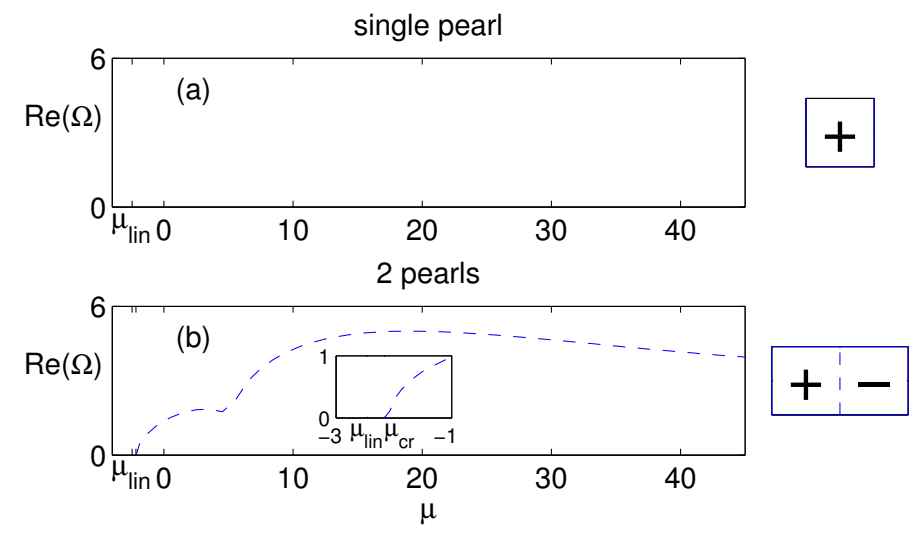

Figure 34: Same as Figure 33 for $\sigma=2$.
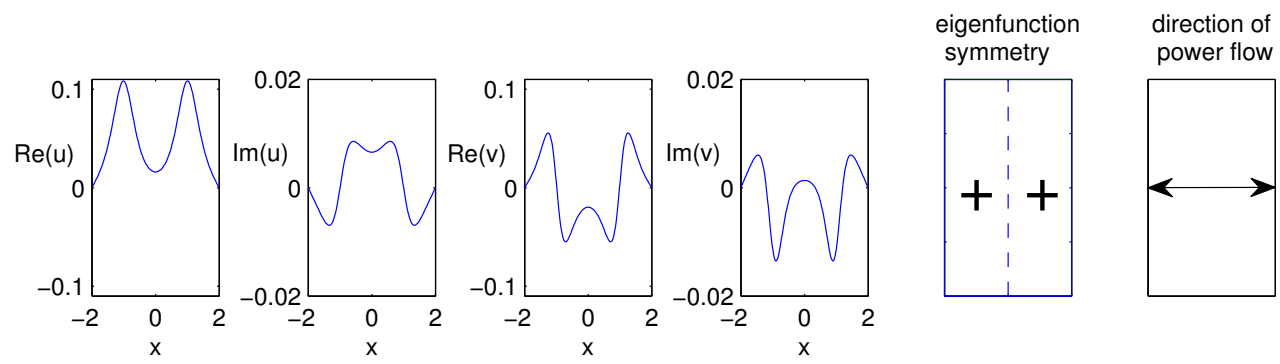

Figure 35: Same as Figure 17 for the one-dimensional NLS with $\sigma=1, D=[-2,2], R=R_{\mu=10}^{(2)}$, and $\Omega_{1}(\mu=10)=0.59+2.2 i$. 

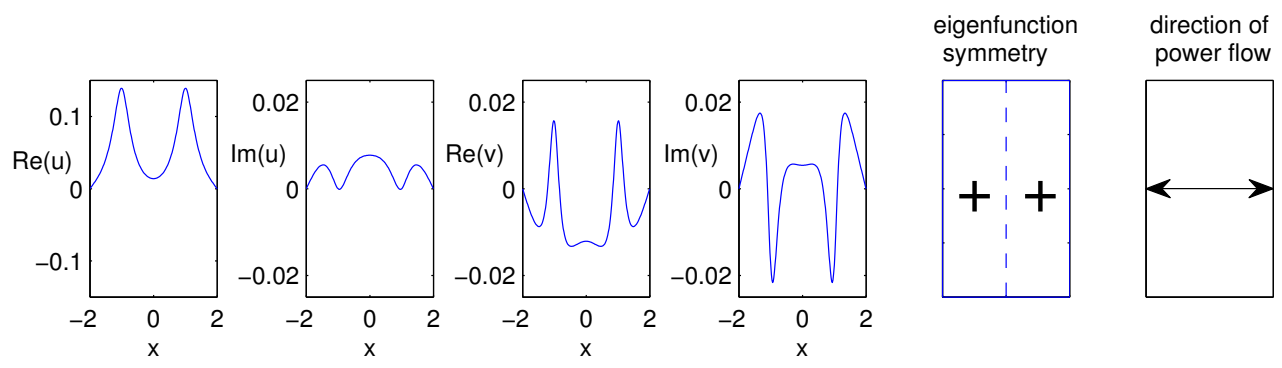

Figure 36: Same as Figure 35 for $\sigma=2$ and $\Omega_{1}(\mu=10)=4.3+5.4 i$.

\section{Numerical methods}

The NLS (3) was integrated numerically using standard fourth-order finite differencing in $\mathbf{x}$, with an implicit Crank-Nicolson method in $z$ for the linear part, and a predictorcorrector method for the nonlinear part. The eigenvalues and eigenvectors of (31) were calculated using Matlab's eig function. The only numerical element which required a nonstandard approach was the calculation of the solitary necklace profiles.

\subsection{Non-spectral renormalization method}

A popular method for calculating multidimensional solitary waves is Petviashvili's spectral renormalization method $(21 ; 22)$. This method, however, can only compute positive solutions, which is not the case with necklace solutions. Therefore, we use this method to compute a single pearl (which is positive), and then "replicate" it into a necklace, as explained in Section 4. In addition, since Petviashvili's method is based on iterations in Fourier space, it cannot be applied on bounded domains. Therefore, following (23), we employ a non-spectral renormalization method, as follows. ${ }^{19}$ We first rewrite (4) as

$$
L(R)=|R|^{2} R, \quad(x, y) \in D, \quad L:=-\Delta+\mu,
$$

subject to $R=0$ on $\partial D$. Then we consider the fixed-point iterations

$$
R_{k+1}=L^{-1}\left[\left|R_{k}\right|^{2} R_{k}\right], \quad k=0,1, \ldots
$$

subject to $R=0$ on $\partial D$, where $R_{k}=R_{k}(x, y)$ is the $k$ th iteration. To implement (37), $L$ is discretized using finite differences and inverted (once) using the LU decomposition. We observe numerically that the iterations (37) diverge to zero for a small initial guess $R_{0}=$ $R_{0}(x, y)$, and to infinity for a large initial guess. To avoid this divergence, we renormalize the solution at each iteration, so that it satisfies the integral relation ${ }^{20},{ }^{21}$

$$
\mathrm{SL}[R]=\mathrm{SR}[R], \quad \mathrm{SL}[R]:=\langle R, R\rangle, \quad \mathrm{SR}[R]:=\left\langle R, L^{-1}\left(|R|^{2} R\right)\right\rangle,
$$

\footnotetext{
${ }^{19}$ For additional inverse iteration methods, see e.g., (24; 25$)$.

${ }^{20}$ Other integral relations can also be used.

${ }^{21}$ Here, $\langle f, g\rangle:=\int f^{*} g d x d y$ denotes the standard inner product.
} 
which is obtained from multiplication of (37) by $R$ and integration over $(x, y)$. Thus, we define $R_{k+\frac{1}{2}}:=c_{k} R_{k}$, where $c_{k}$ is chosen so that $R_{k+\frac{1}{2}}$ satisfies (38), i.e.,

$$
c_{k}^{2} \mathrm{SL}\left[R_{k}\right]=c_{k}^{4} \mathrm{SR}\left[R_{k}\right]
$$

Consequently, $c_{k}=\left(\mathrm{SL}\left[R_{k}\right] / \mathrm{SR}\left[R_{k}\right]\right)^{\frac{1}{2}}$. Therefore, the non-spectral renormalization method reads

$$
R_{k+1}=L^{-1}\left[\left|R_{k+\frac{1}{2}}\right|^{2} R_{k+\frac{1}{2}}\right]=\left(\frac{\left\langle R_{k}, R_{k}\right\rangle}{\left\langle R_{k}, L^{-1}\left(\left|R_{k}\right|^{2} R_{k}\right)\right\rangle}\right)^{\frac{3}{2}} L^{-1}\left[\left|R_{k}\right|^{2} R_{k}\right], \quad k=0,1, \ldots
$$

To illustrate the convergence of this method, consider the iterations (39) when $D$ is the square $[-1,1]^{2}, \mu=1$, the initial guess is $R_{0}=\sin \left(\pi\left(\frac{x-1}{2}\right)\right) \sin \left(\pi\left(\frac{y-1}{2}\right)\right), L$ is discretized using fourth-order center-difference scheme, and $d x=d y=0.05$. Fig. 37(a) shows that after 40 iterations $c_{k}$ converges to 1 with machine accuracy, thus confirming the convergence of the iterations. In order to check that the iterations converge to a solitary wave, we recall that the Pohozaev identities on a bounded domain read

$$
\begin{aligned}
\mu\left\|R_{\mu}\right\|_{2}^{2} & =\frac{1}{2}\left\|R_{\mu}\right\|_{4}^{4}-\frac{1}{2} \int_{\partial D}(\mathbf{x} \cdot \mathbf{n})\left(\nabla R_{\mu} \cdot \mathbf{n}\right)^{2} d \mathbf{s} \\
\left\|\nabla R_{\mu}\right\|_{2}^{2} & =\frac{1}{2}\left\|R_{\mu}\right\|_{4}^{4}+\frac{1}{2} \int_{\partial D}(\mathbf{x} \cdot \mathbf{n})\left(\nabla R_{\mu} \cdot \mathbf{n}\right)^{2} d \mathbf{s}
\end{aligned}
$$

where $\mathbf{n}$ is the outward unit normal to $\partial D$. To avoid computing boundary integrals, we check whether $R_{k}$ satisfies the sum of these Pohozaev identities, i.e., whether $E_{\text {Pohozaev }}\left(R_{k}\right) \rightarrow 0$, where

$$
E_{\text {Pohozaev }}(R):=\mu\|R\|_{2}^{2}+\|\nabla R\|_{2}^{2}-\|R\|_{4}^{4} .
$$

Fig. 37(b) shows that $E_{\text {Pohozaev }}$ converges to $O\left(10^{-3}\right)$, and not to machine accuracy. This is because the iterations converge to a solution of a discretized version of (4). In other words, the $O\left(10^{-3}\right)$ error of the Pohozaev identities is determined by the discretization error and not by the convergence of the iterations. Indeed, we verified that if we use smaller values of $d x$ and $d y$, the limiting Pohozaev error reduces according to the order of the discretization scheme being used.
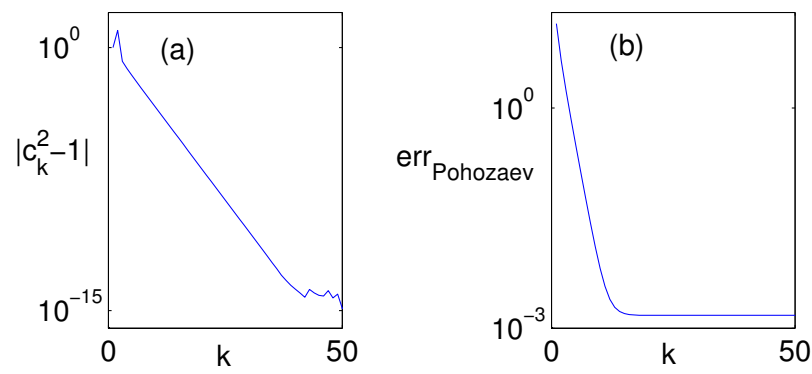

Figure 37: Convergence of the non-spectral renormalization method. (a) $\left|c_{k}^{2}-1\right|$ as a function of the iteration number $k$. (b) $\operatorname{err}_{\text {Pohozaev }}\left(R_{k}\right)$ as a function of the iteration number. 
Acknowledgments We thank A. Gaeta, B. Ilan, N. Fusco, and Y. Pinchover for useful suggestions. This research was partially supported by grant \#177/13 from the Israel Science Foundation (ISF), and by the Kinetic Research Network (KI-Net) under the NSF Grant No. RNMS \#1107444.

[1] G. Fibich, The Nonlinear Schrödinger Equation, Springer, New-York, 2015.

[2] M. Soljacić, S. Sears, M. Segev, Self-trapping of necklace beams in self-focusing Kerr media, Phys. Rev. Lett. 81 (1998) 4851-4854.

[3] T. Grow, A. Ishaaya, L. Vuong, A. Gaeta, Collapse and stability of necklace beams in Kerr media, Phys. Rev. Lett. 99 (2007) 133902.

[4] A. S. Desyatnikov, Y. S. Kivshar, Necklace-ring vector solitons, Phys. Rev. Lett. 87 (2001) 033901.

[5] Y. Kartashov, B. Malomed, V. Vysloukh, L. Torner, Stabilization of multibeam necklace solitons in circular arrays with spatially modulated nonlinearity, Phys. Rev. A 80 (2009) 053816 .

[6] M. Soljacić, M. Segev, Integer and fractional angular momentum borne on self-trapped necklace-ring beams, Phys. Rev. Lett. 86 (2001) 420-423.

[7] J. Yang, I. Makasyuk, P. G. Kevrekidis, H. Martin, B. A. Malomed, D. J. Frantzeskakis, Z. Chen, Necklacelike solitons in optically induced photonic lattices, Phys. Rev. Lett. 94 (2005) 113902.

[8] N. Jhajj, E. Rosenthal, R. Birnbaum, J. Wahlstrand, H. Milchberg, Demonstration of long-lived high-power optical waveguides in air, Phys. Rev. X 4 (2014) 011027.

[9] G. Fibich, F. Merle, Self-focusing on bounded domains, Physica D 155 (2001) 132-158.

[10] R. Fukuizumi, F. Selem, H. Kikuchi, Stationary problem related to the nonlinear Schrödinger equation on the unit ball, Nonlinearity 25 (2012) 2271-2301.

[11] B. Noris, H. Tavares, G. Verzini, Existence and orbital stability of the ground states with prescribed mass for the $L^{2}$-critical and supercritical NLS on bounded domains, Analysis \& PDE.

[12] E. Kirr, P. Kevrekidis, E. Shlizermans, M. Weinstein, Symmetry-breaking bifurcation in nonlinear Schrödinger/Gross-Pitaevskii equations, SIAM J. Math. Anal. 40 (2008) 566604 .

[13] B. Ilan, Y. Sivan, G. Fibich, A quantitative approach to soliton instability, Opt. Lett. 36 (2011) 397-399.

[14] Y. Sivan, G. Fibich, B. Ilan, Drift instability and tunneling of lattice solitons, Phys. Rev. E 77 (2008) 045601(R). 
[15] Y. Sivan, G. Fibich, B. Ilan, M. Weinstein, Qualitative and quantitative analysis of stability and instability dynamics of positive lattice solitons, Phys. Rev. E 78 (2008) 046602 .

[16] G. Fibich, N. Gavish, Theory of singular vortex solutions of the nonlinear Schrödinger equation, Physica D 237 (2008) 2696-2730.

[17] A. Cianchi, N. Fusco, Steiner symmetric extremals type inequalities, Advances in Mathematics 203 (2006) 673-728.

[18] G. Fibich, A. Gaeta, Critical power for self-focusing in bulk media and in hollow waveguides, Opt. Lett. 25 (2000) 335-337.

[19] N. Vakhitov, A. Kolokolov, Stationary solutions of the wave equation in a medium with nonlinearity saturation, Radiophys. Quant. Elec. 16 (1973) 783-789.

[20] G. Fibich, Y. Sivan, M. Weinstein, Bound states of nonlinear Schrödinger equations with a periodic nonlinear microstructure, Physica D 217 (2006) 3157.

[21] V. Petviashvili, Equation of an extraordinary soliton, Sov. J. Plasma Phys. 2 (1976) $257-258$.

[22] M. Ablowitz, Z. Musslimani, Spectral renormalization method for computing selflocalized solutions to nonlinear systems, Opt. Lett. 30 (2005) 2140-2142.

[23] G. Baruch, G. Fibich, N. Gavish, Singular standing ring solutions of nonlinear partial differential equations, Physica D 239 (2010) 1968-1983.

[24] K. Georg, On the convergence of an inverse iteration method for nonlinear elliptic eigenvalue problems, Numer. Math. 32 (1979) 69-74.

[25] J. Descloux, J. Rappaz, A nonlinear inverse power method with shift, SIAM J. Numer. Anal. 20 (1983) 1147-1152. 